\title{
Submesoscale Eddy Vertical Covariances and Dynamical Constraints from High-Resolution Numerical Simulations
}

\author{
Joseph M. D’Addezio, GregG A. JACOBS, And MAX YAREMchuK \\ Ocean Dynamics and Prediction, Naval Research Laboratory, Stennis Space Center, Mississippi \\ INNOCENT SOUOPGUI \\ Department of Physics, University of New Orleans, New Orleans, Louisiana
}

(Manuscript received 26 April 2019, in final form 2 January 2020)

\begin{abstract}
We analyze high-resolution $(1 \mathrm{~km})$ simulations of the western Pacific, Gulf of Mexico, and Arabian Sea to understand submesoscale eddy dynamics. A mask based on the Okubo-Weiss parameter isolates small-scale eddies, and we further classify those with $|\zeta / f| \geq 1$ as being submesoscale eddies. Cyclonic submesoscale eddies exhibit a vertical depth structure in which temperature anomalies from the large-scale background are negative. Peak density anomalies associated with cyclonic submesoscale eddies are found at a depth approximately twice the mixed layer depth (MLD). Within anticyclonic submesoscale eddies, temperature anomalies are positive and have peak density anomalies at the MLD. The depth-depth covariance structure for the cyclonic and anticyclonic submesoscale eddies have maxima over a shallow region near the surface and weak off diagonal elements. The observed vertical structure suggests that submesoscale eddies have a shallower depth profile and smaller vertical correlation scales when compared to the mesoscale phenomenon. We test a twodimensional submesoscale eddy dynamical balance. Compared to a geostrophic dynamical balance using only pressure gradient and Coriolis force, including velocity tendency and advection produces lower errors by about $20 \%$. In regions with strong tides and associated internal waves (western Pacific and Arabian Sea), using the mixed layer integrated small-scale steric height within the dynamical equations produces the lowest magnitude errors. In areas with weak tides (Gulf of Mexico), using small-scale sea surface height (SSH) produces the lowest magnitude errors. Recovering a submesoscale eddy with the correct magnitude and rotation requires integration of small-scale specific volume anomalies well below the mixed layer.
\end{abstract}

\section{Introduction}

The forthcoming wide-swath Surface Water Ocean Topography (SWOT) satellite mission (Fu and Ubelmann 2014) is expected to resolve sea surface height (SSH) features associated with mesoscale, submesoscale, and internal wave activity on a global scale. Historically, ocean prediction efforts have focused on the mesoscale due to observational constraints. Nadir altimeters can only resolve phenomena with wavelengths of approximately $100 \mathrm{~km}$ and greater (Ducet et al. 2000; Fu and Ubelmann 2014). The new SWOT capabilities motivate a deeper understanding of smaller-scale dynamics and how ocean models can best assimilate surface height expressions of phenomena smaller than mesoscale.

Corresponding author: Joseph M. D’Addezio, joseph.daddezio@ nrlssc.navy.mil
Variational data assimilation schemes are heavily dependent upon decorrelation length scales that spread observation innovations based on the Rossby deformation length scale (Cummings 2005). This technique has strong utility when considering only mesoscale observations provided by nadir altimeters. However, the approach becomes problematic when assimilating data that contain a larger set of scale-dependent dynamics, as with SWOT, because using a single decorrelation length scale is known to bias errors depending on the scale ( $\mathrm{Li}$ et al. 2015a; D'Addezio et al. 2019). If one chooses a relatively large decorrelation length scale, the assimilation preferentially corrects the largest of scales, and small-scale information is lost. If a shorter decorrelation length scale is used, the assimilation neglects the large-scale mesoscale field and initialization errors propagate downscale during forward integration. A multistep analysis procedure addresses this problem by 
treating each scale successively. Li et al. (2015b) demonstrate such approaches successfully reduce bulk errors in forecast models that explicitly resolve submesoscale dynamics.

In addition to the range of feature sizes, multiscale assimilation systems must consider the continuum of dynamics contained within the high-resolution observations that instruments like SWOT will provide. Two types of dynamics dominate the large- and small-scale surface elevation features that SWOT will observe. First are the eddying features. Mesoscale eddies, with small Rossby number, are geostrophically balanced to a high degree. Submesoscale eddies, however, with Rossby number of unity or larger primarily obey gradient wind balance (Capet et al. 2008b): a balance between planetary vorticity, pressure, and advection. Second are internal gravity waves that generate ageostrophic surface expressions as oscillations of the internal isopycnals change thickness and thus steric content (Chavanne et al.2010). The prediction community requires a set of methodologies to accurately assimilate all of these disparate physical phenomena into ocean models in order to generate accurate forecasts. Therefore, the impetus for this study is to develop statistics on the vertical and horizontal dynamics that govern submesoscale eddy processes to aid the development of a more sophisticated, scale-dependent background error covariance for implementation within a multiscale assimilation system.

To further this objective, we simulate three large ocean regions at high horizontal resolution $(1 \mathrm{~km})$ : the western Pacific, Gulf of Mexico, and Arabian Sea. A spatial filter, based on the regional Rossby radius of deformation, separates "large-scale" and "small-scale" fields. The Okubo-Weiss parameter applied to the small-scale field identifies small-scale eddies. "Submesoscale" eddies are defined as smallscale eddies with surface vorticity normalized by the Coriolis parameter greater than or equal to one: $|\zeta / f| \geq 1$, the term being a Rossby number frequently used for dynamically defining the submesoscale (McWilliams 2016; D'Asaro et al. 2018). Current understanding suggests that submesoscale eddies have large variability within the mixed layer (e.g., Thompson and Lazar 2016; Bachman et al. 2017). Recent observational evidence extends this hypothesis. In situ data collected and analyzed by $\mathrm{Yu}$ et al. (2019) demonstrated submesoscale eddy vorticity penetration up to $200 \mathrm{~m}$ below the mixed layer. The mean vertical structure of our modeled submesoscale eddies is consistent with Yu et al. (2019), whereby we observe density anomalies well below the base of the mixed layer. An example illustrates this (Fig. 1). In the Arabian Sea during January, a cyclonic submesoscale eddy with $\zeta / f>2$ propagates around an anticyclonic mesoscale eddy (Fig. 1a). A vertical slice of density through both eddies shows a large-scale isopycnal displacement toward the ocean bottom with a local surfaceward displacement in the proximity of the submesoscale eddy (Fig. 1b). The largescale filtered field highlights the mesoscale eddy downward isopycnal bending, which extends below $500 \mathrm{~m}$ (Fig. 1c). Finally, the small-scale density anomalies associated with the submesoscale eddy are absent from the mixed layer and are instead concentrated 50-200 $\mathrm{m}$ below the base of the mixed layer (Fig. 1d). This model snapshot provides an illustration of the statistics we present throughout this paper, which suggest that submesoscale eddies have significant baroclinic effects below the mixed layer and that the submesoscale eddy vertical structures are significantly different from mesoscale variations.

At the surface, we hypothesize that submesoscale eddy currents require more than just the geostrophic balance to accurately describe the forces in a Eulerian coordinate. The force balance we propose for the submesoscale eddies is inappropriate for wave dynamics, which is an important dynamical regime that SWOT will observe along with the eddy dynamics. Barotropic tides force internal gravity waves with tidal frequencies that have surface elevation expressions similar in horizontal scale to submesoscale eddies. Therefore, the experiments here also explore how the superposition of mesoscale, submesoscale, and internal wave contributions to SSH affect the potential SWOT observations. In the following paragraphs, we briefly review mesoscale, submesoscale, and internal gravity wave dynamics and conclude the section by describing how the paper is organized.

Submesoscale ocean eddies inhabit a unique space in the continuum of ocean dynamics between large mesoscale eddies and small-scale turbulence. Mesoscale eddies derive energy from available potential energy within geostrophically balanced horizontal and vertical shear along boundary currents as well as the ocean interior. The baroclinic instabilities have horizontal scales on the order of the Rossby radius of deformation, and mesoscale vertical influence extends throughout the main pycnocline (Cummings et al. 2009; Helber et al. 2013). These mesoscale currents pass kinetic energy to the submesoscale eddies, which are known to both cascade energy downscale, and also pass energy back upscale; although current understanding suggests that the former is the dominant effect, whereby submesoscale currents provide an important route toward energy dissipation at smaller scales (Boccaletti et al. 2007; Capet et al. 2008b; McWilliams 

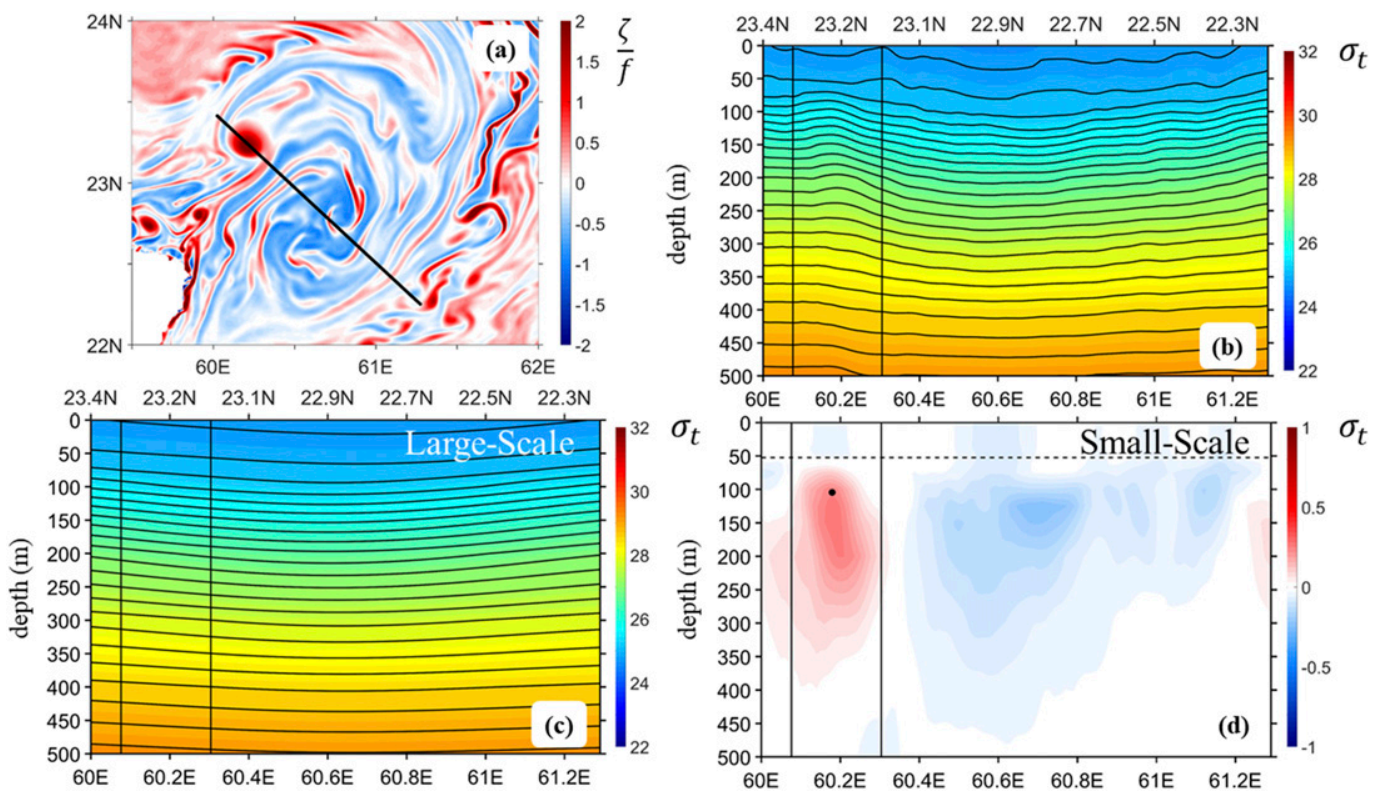

FIG. 1. Example cyclonic submesoscale eddy propagating around an anticyclonic mesoscale eddy in the Arabian Sea during January. (a) Surface vorticity normalized by the Coriolis parameter with a marked cross section through the submesoscale and mesoscale eddies. (b) Vertical cross section of density $\left(\sigma_{t}=\sigma-1000\right)\left(\mathrm{kg} \mathrm{m}^{-3}\right)$ through the line marked in (a). The vertical black bars denote the approximate latitude/longitude bounds of the submesoscale eddy. (c) The same cross section from (b), but for the large-scale density field. The large-scale field was obtained by smoothing (b) at each depth using a 2D spatial filter. (d) As in (c), but for the small-scale density field. The smallscale field was obtained by subtracting (c) from (b). The dashed, horizontal line is the area-averaged MLD. The black dot within the submesoscale eddy denotes the depth 2 times greater than the MLD.

2016). Submesoscale eddies have horizontal scales on the order of several kilometers and are thought to have vertical variability mainly within the mixed layer (e.g., Thompson and Lazar 2016; Bachman et al. 2017). Mesoscale eddies have relatively long time scales (weeks) and are thus reasonably approximated by the steady-state geostrophic balance. Submesoscale eddies are more transient (days) making the geostrophic approximation less useful (Thomas et al. 2008; Capet et al. 2008b; McWilliams 2016). Additionally, submesoscale features are known to be strongly advective in the vertical, allowing the transfer of energy and mass between the surface and subsurface environments (Klein and Lapeyre 2009; Koszalka et al. 2009; Zhong and Bracco 2013; Lévy et al. 2012; Brannigan 2016; Zhong et al. 2017; Su et al. 2018; Lévy et al. 2018). The mesoscale phenomena, however, typically induce strong horizontal stirring but have weak vertical velocities (Capet et al. 2008a). Thus, mesoscale and submesoscale eddies are dynamically distinct and not just different in characteristic length scales.

The physics of the two eddy regimes stand in contrast with those of the internal gravity wave dynamics. Internal waves have near-inertial and tidal frequencies set by the phenomena that predominantly generate them: winds and tides. They create isopycnals displacements with amplitude $O(10) \mathrm{m}$, have phase speeds that depend on vertical stratification, and have spatial scales that range from $100 \mathrm{~m}$ to $100 \mathrm{~km}$ (Levine 1983; St. Laurent et al. 2012). The combination of mesoscale, submesoscale, and internal wave signals imposed on surface elevation is a challenge for understanding and using SWOT SSH observations. Therefore, an important part of the experimentation presented here is addressing how internal wave surface expressions affect the utility of dynamical balances that target the submesoscale eddy phenomenon.

Section 2 describes the modeling framework as well as the methodologies used for separation of the largeand small-scale fields, eddy identification of smallscale eddies, and identification of submesoscale eddies. Section 3 presents the results of the data analysis by demonstrating the differences in large-scale and submesoscale eddy vertical structures and then by evaluating errors with respect to a sequence of increasingly complex dynamics applied to small-scale surface elevation and two-dimensional velocity within submesoscale eddies. The paper concludes by summarizing the findings 

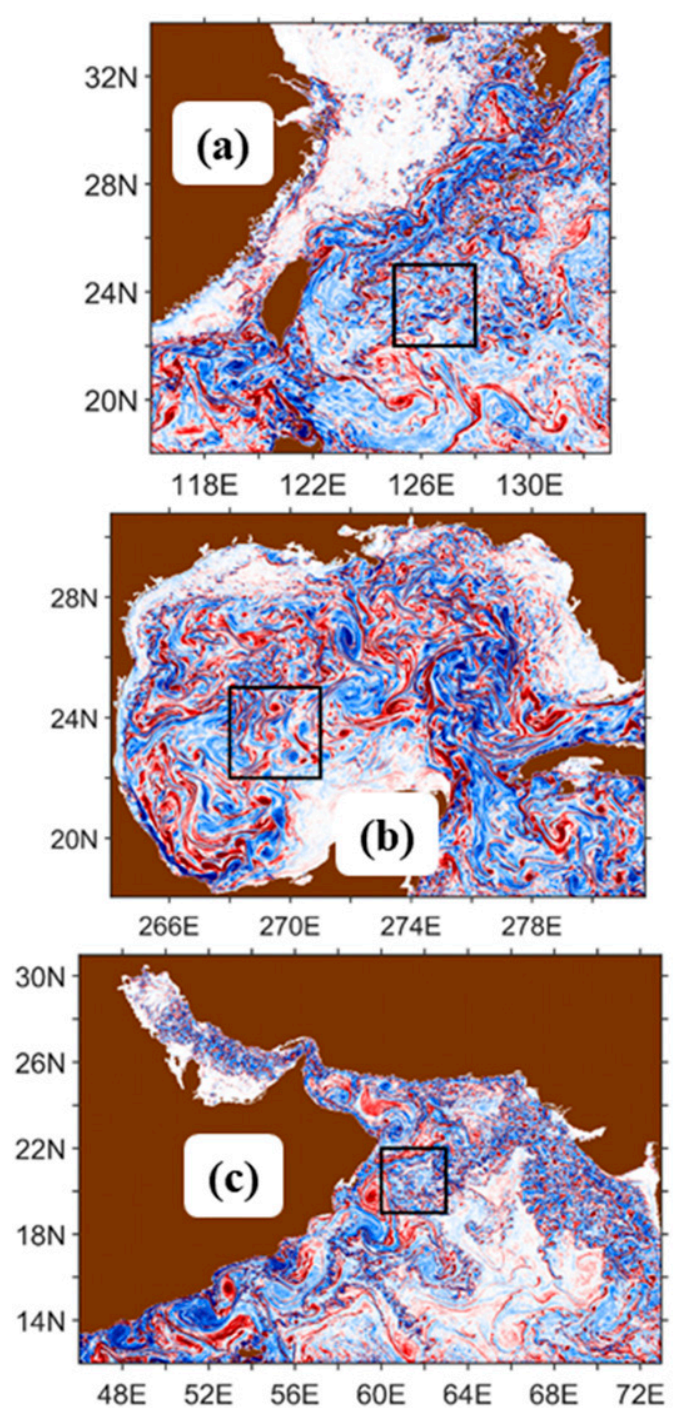

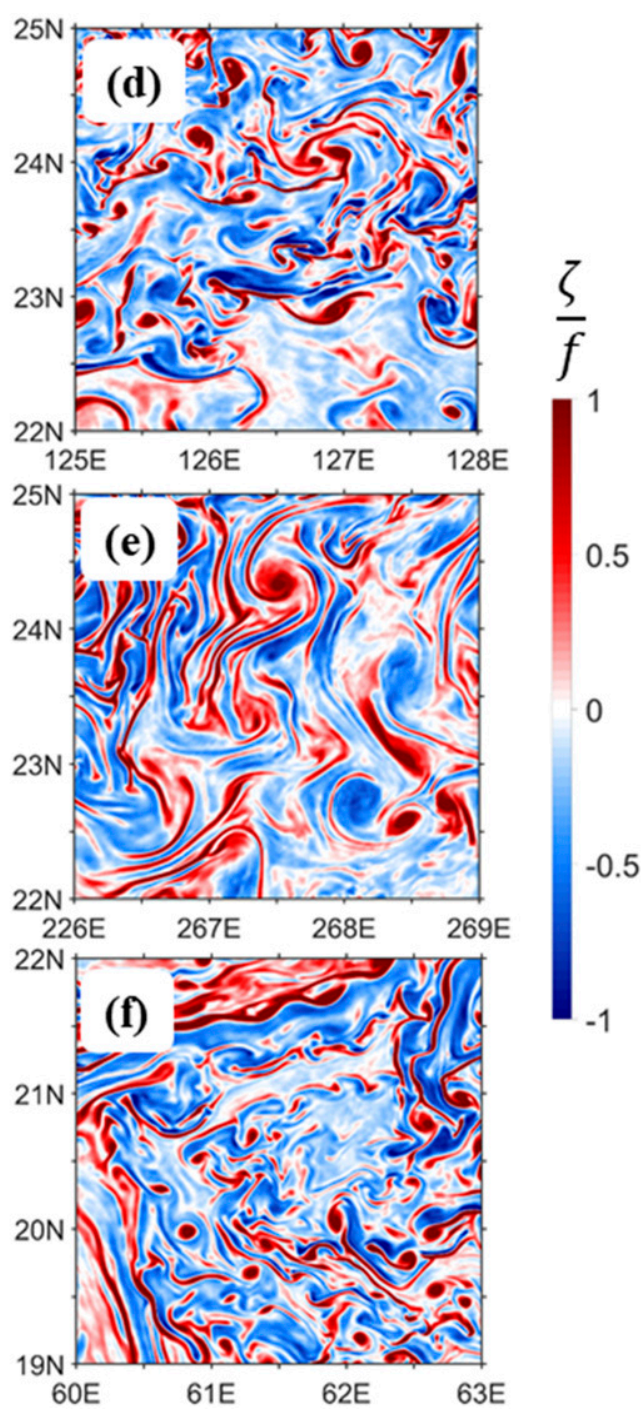

0.5

$-0.5$

FIG. 2. Snapshot (1 Jan 2016) of model surface vorticity normalized by the Coriolis parameter in the (a) western Pacific, (b) Gulf of Mexico, and (c) Arabian Sea. (d)-(f) Zoomed in subsections of each region (black boxes) highlighting the small-scale features present in the simulations. and discussing the potential utility of the results for ocean prediction in section 4 .

\section{Methods}

\section{a. Modeling}

This study uses numerical simulations in three distinct regions of the global ocean: the western Pacific, Gulf of Mexico, and Arabian Sea (Fig. 2). The western Pacific is a highly dynamic region featuring the strong Kuroshio western boundary current, driving ubiquitous mesoscale and submesoscale variability in addition to the mesoscale and submesoscale field within the interior of the subtropical recirculation gyre. Barotropic tides interact with the sill in the Luzon Strait, the shelf break along the
Yellow Sea, and other topographic features to generate internal tides that propagate into the open ocean. The Gulf of Mexico features the Loop Current circulation driving strong horizontal velocities and shedding large mesoscale eddies into the interior of the semi-enclosed basin. In contrast with the western Pacific, the Gulf of Mexico has weak tidal signal that produces less intense internal tides. This region is useful for understanding the relative impacts of internal waves on the viability of using the proposed dynamical relations on observed and modeled SSH. Finally, the Arabian Sea does not have a western boundary current feature like the other two regions, but it does have strong mesoscale activity, and tides interact with the shelf break to produce large internal waves that propagate into the open ocean. 
TABLE 1. Overview of the modeling framework used for each geographic region.

\begin{tabular}{|c|c|c|c|}
\hline & Western Pacific & Gulf of Mexico & Arabian Sea \\
\hline \multicolumn{4}{|c|}{ Ocean model } \\
\hline Model & \multicolumn{3}{|c|}{ NCOM (Barron et al. 2006) } \\
\hline \multirow{2}{*}{ Geographic bounds (Fig. 2) } & $116^{\circ}-133^{\circ} \mathrm{E}$ & $262^{\circ}-280^{\circ} \mathrm{E}$ & $46^{\circ}-73^{\circ} \mathrm{E}$ \\
\hline & $18^{\circ}-34^{\circ} \mathrm{N}$ & $18^{\circ}-31^{\circ} \mathrm{N}$ & $12^{\circ}-31^{\circ} \mathrm{N}$ \\
\hline Horizontal resolution & $1 \mathrm{~km}$ & $1 \mathrm{~km}$ & $1 \mathrm{~km}$ \\
\hline Vertical layers & 50 & 50 & 50 \\
\hline Boundary conditions & 3-km NCOM & $1 / 12^{\circ} \mathrm{HYCOM}$ & 3-km NCOM \\
\hline Assimilation & No & Yes & No \\
\hline \multicolumn{4}{|c|}{ Atmospheric Forcing } \\
\hline Model & NAVGEM (Hogan et al. 2014) & COAMPS (Hodur 1997) & NAVGEM (Hogan et al. 2014) \\
\hline Surface wind stress & On & On & On \\
\hline Latent heat flux & On & On & On \\
\hline Sensible heat flux & On & On & On \\
\hline Precipitation & On & Off & On \\
\hline Model & \multicolumn{3}{|c|}{$\begin{array}{l}\text { River inflow } \\
\text { Climatological river database (Barron and Smedstad 2002) }\end{array}$} \\
\hline Model & \multicolumn{3}{|c|}{ Tidal forcing } \\
\hline
\end{tabular}

Each domain also has different large-scale vertical stratification that affects the thermocline depth, the mesoscale vertical structure, the mixed layer depth (MLD), and the depth structure of submesoscale features. The unique set of interacting dynamics enclosed within each region will allow us to compare and contrast the submesoscale variability found in each domain. Table 1 provides a description of the different model parameters used to simulate each region. The remainder of this section provides a detailed discussion of the summary in Table 1.

The Navy Coastal Ocean Model (NCOM; Barron et al. 2006) provides the numerical representation of ocean dynamics. NCOM integrates the primitive equations forward in time using Boussinesq and hydrostatic assumptions on a regular grid in the horizontal and a hybrid $\sigma / z$ grid in the vertical. The vertical grid structure allows terrain following capabilities in shallow waters and enhanced representation of mixed layer dynamics in the open ocean. The setups for the domains all use 50 vertical layers with an approximately 1 -m-thick surface sigma layer. Layer thicknesses increase steadily to the maximum model depth of $4000 \mathrm{~m}$. The horizontal resolution in all domains is $1 \mathrm{~km}$. This is an important specification as this resolution allows the simulation to permit submesoscale eddies with spatial scales $O(10) \mathrm{km}$ (Capet et al. 2008a).

Lateral boundary conditions were provided by the $1 / 12^{\circ}$ global Hybrid Coordinate Ocean Model (HYCOM; Metzger et al. 2017). For the Gulf of Mexico domain, the interpolated HYCOM solution directly forced the 1-km NCOM grid. This direct solution does not work for the western Pacific and Arabian Sea domains. Numerical instability in the high-resolution domains occurs when interpolating HYCOM boundary conditions going from 9 to $1 \mathrm{~km}$ resolution. The solution to this problem is the introduction of an intermediate NCOM simulation modeled at $3-\mathrm{km}$ horizontal resolution with boundaries at least $3^{\circ}$ larger than the final $1-\mathrm{km}$ domain on all sides using boundary conditions from global HYCOM. The 3-km NCOM simulation provided boundary conditions to the final 1-km NCOM grid. This approach eliminated the instability issues. In a similar fashion, the western Pacific and Arabian Sea simulations used an interpolated initial condition from the intermediary $3-\mathrm{km}$ NCOM simulation, while the Gulf of Mexico used an interpolated initial condition from global HYCOM. Each simulation was spun up for at least one month prior to 1 January 2016, and data were output in 3-h intervals covering the period of 1 January 2016-31 December 2016.

Two sources provided atmospheric forcing: Navy Global Environmental Model (NAVGEM; Hogan et al. 2014) and Coupled Ocean-Atmosphere Mesoscale Prediction System (COAMPS; Hodur 1997). NAVGEM fields provided the momentum and heat fluxes forcing the western Pacific and Arabian Sea simulations, and COAMPS provided fields for forcing the Gulf of Mexico simulation. NAVGEM is a global atmospheric model with a horizontal resolution of approximately $37 \mathrm{~km}$ that outputs data every $3 \mathrm{~h}$. Surface wind stress, atmospheric temperature, atmospheric humidity, wind speed, and precipitation from NAVGEM along with the ocean model SST provided the time-varying surface fluxes. COAMPS is a higher-resolution regional atmospheric model with horizontal grid spacing of $16 \mathrm{~km}$. The data are output in 
(a)

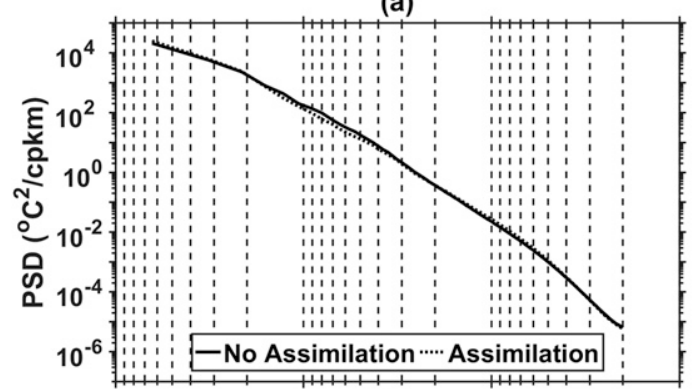

(c)

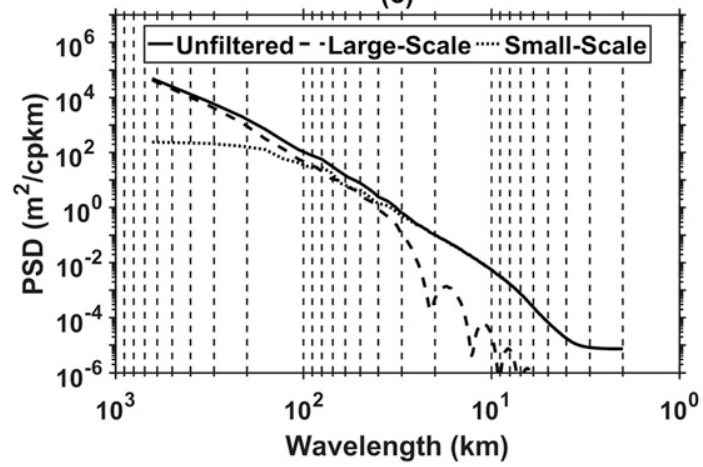

(b)

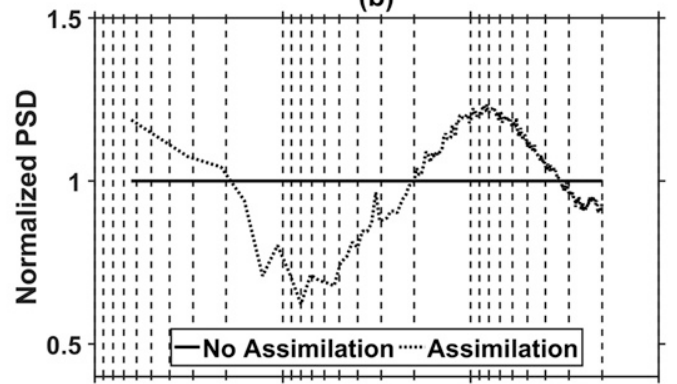

(d)

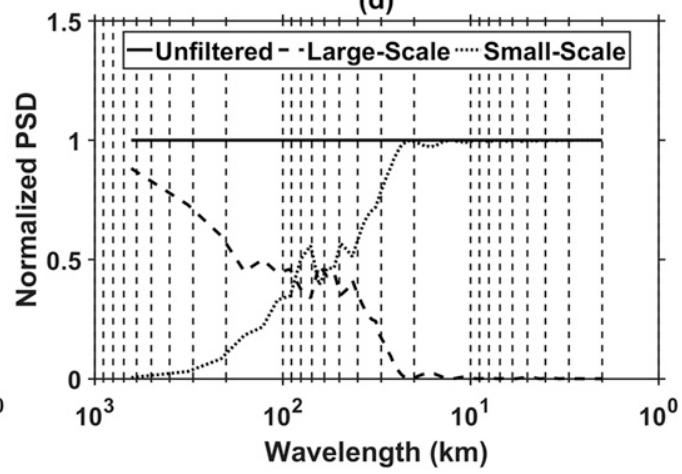

FIG. 3. (a) 100-m temperature wavenumber spectra $\left({ }^{\circ} \mathrm{C}^{2} \mathrm{cpkm}^{-1}\right)$ for a subregion of the western Pacific $\left(125.5^{\circ}-\right.$ $\left.132^{\circ} \mathrm{E}, 20^{\circ}-25.5^{\circ} \mathrm{N}\right)$. The solid black line resulted from a simulation that did not assimilate any observations, while the dashed black line resulted from a simulation that did. (b) Spectra from (a) normalized by the spectrum from the nonassimilative experiment to show the relative energy levels as a function of wavelength. (c) Unfiltered, largescale, and small-scale SSH wavenumber spectra $\left(\mathrm{m}^{2} \mathrm{cpkm}^{-1}\right)$. (d) Spectra from (c) normalized by the unfiltered spectrum.

3-h intervals and the Gulf of Mexico simulation used COAMPS results in a manner similar to NAVGEM.

A climatological river database of monthly mean transport (Barron and Smedstad 2002) provided lateral freshwater flow. The database introduced climatological freshwater input at fixed points that approximate the geographic locations of large river mouths. Tidal currents and water levels from Oregon Tidal Inverse Solution (OTIS; Egbert and Erofeeva 2002) provided lateral forcing in addition to the global HYCOM solutions. Tidal potential is also included in the model forcing. In a post processing procedure, we conducted a harmonic analysis to remove the barotropic and stationary baroclinic tides from SSH and velocity. However, this methodology does not remove nonstationary baroclinic tides due to the mesoscale field changing ocean stratification controlling internal wave speed and thus altering internal tide phase (Shriver et al. 2014).

The western Pacific and Arabian Sea simulations did not assimilate observations, while the Gulf of Mexico simulation did. The assimilation scheme focuses on relatively large-scale features and makes mesoscale assumptions within the data assimilation analysis
(Cummings 2005). One might hypothesize that the mesoscale focused assimilation could suppress some of the small-scale variability we are trying to quantify. This possibility was tested by analyzing the wavenumber spectra of two parallel simulations, one being a free running simulation and the other including data assimilation. Two-dimensional power spectral density (PSD) was calculated over a subregion of the western Pacific $\left(125.5^{\circ}-132^{\circ} \mathrm{E}, 20^{\circ}-25.5^{\circ} \mathrm{N}\right)$ every $3 \mathrm{~h}$ for one month. The ensemble of PSD was then averaged in time. Finally, a one-dimensional spectrum was produced by azimuthally averaging the time-averaged PSD (Richman et al. 2012). The results are shown in Fig. 3. Figure 3a shows that PSD from each simulation is similar. Figure $3 \mathrm{~b}$ shows the assimilative spectrum normalized by the spectrum from the nonassimilative simulation. The normalized spectrum suggests that the assimilation reduced spatial variability in the mesoscale band $(300-40 \mathrm{~km})$. Below $20 \mathrm{~km}$, the simulation using data assimilation has greater variability than the free running simulation. This result suggests that data assimilation is not substantially affecting the submesoscale features we are targeting in this experiment. 


\section{b. Data analysis}

\section{1) SCALE SEPARATiON}

The example in Fig. 1 indicates a simple method to initially separate large- and small-scale features by filtering. The filtering itself does not identify submesoscale eddies. The identification of submesoscale eddies in the filtered fields and subsequent examination of vertical structure and dynamics within the submesoscale eddies are discussed in later sections. In this section, we separate a model field $X$ (e.g., SSH or velocity at a certain depth level) into large-scale $X_{L}$ and small-scale $X_{S}$ constituents defined as

$$
X_{L}=F(X) ; X_{S}=X-F(X),
$$

where $F$ is a filter described below. Capet et al. (2008a) removed a long-term mean to partition their large- and small-scale fields. In section $3 \mathrm{~d}(1)$, we describe a twodimensional dynamical relation that involves advection of the small-scale field by the large-scale phenomena. Removing the long-term mean from the large-scale velocity field can yield an inaccurate representation of the advection term because the background synoptic current may be an important component of smaller-scale motion. Therefore, we examine the deviations from the local largescale features to help diagnose small-scale processes.

Ocean variables have power spectra that have no clear breaks (Fig. 3a). This makes it challenging to separate scales using one set of spatial and/or temporal parameters. Thus, we rely on a simple set of assumptions to isolate the small-scale regime. The Rossby radius of deformation approximates the spatial scale of the geostrophically balanced dynamics. Each of the three regions under consideration occupy a similar latitudinal band (Fig. 2), the deformation radius being primarily a function of the Coriolis parameter, dependent on latitude, with some variation due to local stratification. For each of the three regions, the first baroclinic mode deformation radius is approximately $50 \mathrm{~km}$ (Chelton et al. 1998). Therefore, our low-pass filter $F$ is a Gaussianshaped smoothing kernel with a half-width (i.e., horizontal length scale) of 50 grid points $(\sim 50 \mathrm{~km})$ from the centroid of the filter. Evaluated in one dimension, the filter can be expressed as

$$
F_{i j}=\exp \left[-\frac{\left(x_{i}-x_{j}\right)^{2}}{2 l_{x}^{2}}\right],
$$

where $x_{i}$ and $x_{j}$ are coordinates along the single dimension and $l_{x}$ is the half-width of the filter (Yaremchuk et al. 2013). The filter assumes that land points are zero, introducing a bias at the boundaries. The effects of this assumption are minimized in the analyses (section 3 ) by excluding grid points with water depths shallower than $1000 \mathrm{~m}$. Wavenumber spectra of the unfiltered, large-scale, and small-scale western Pacific SSH are presented in Fig. 3c. Normalizing by the unfiltered spectrum shows the crossing point between the largeand small-scale spectra at approximately $50 \mathrm{~km}$ (Fig. 3d). Thus, the filter performs as anticipated on the twodimensional geophysical parameters to which it is applied.

An example of separating the large- and small-scale fields within SSH per Eq. (1) is provided in Fig. 4. The resulting large-scale fields feature familiar characteristics. The Kuroshio boundary current is evident in the western Pacific, the Loop Current in the Gulf of Mexico, and a chain of cyclonic and anticyclonic mesoscale eddies along the Arabian Peninsula in the Arabian Sea. The small-scale fields feature an array of eddy features entangled with wave interference patterns particularly in the western Pacific and Arabian Sea. The wave patterns are not as evident in the Gulf of Mexico, which is expected as the weak tidal forcing in the region produces much lower magnitude internal tides. Some effects of the mesoscale features persist in the small-scale field. This is inevitable when using a spatial filter because ocean horizontal wavenumber spectra do not have clear breaks (Fig. 3). A combination of spatial and temporal filtering was explored. Large- and smallscale features do tend to have more distinct separation in time, but experiments spatially filtering a centered 2-day mean field, as was done by Capet et al. (2008a), did not produce a substantially different result than that demonstrated in Fig. 4 (these results are not shown). Bachman et al. (2017) also recently employed an approach that relied on spatial filtering alone. Therefore, we proceed with results based on the separation by spatial filtering.

Mesoscale features dominate the large-scale field, though Fig. $3 \mathrm{c}$ demonstrates that because the spectrum of the small-scale field is red, the small-scale field still contains a significant amount of long wavelength variability. Therefore, we cannot simply call our small-scale field "submesoscale" because some larger-scale features have leaked into the fields. The next section describes an eddy selection criterion used to isolate submesoscale eddies in the small-scale field.

\section{2) EDDY IDENTIFICATION}

Figure 4 shows a separation of features, and we must determine if different dynamics predominate in submesoscale eddies. The small-scale field contains influence from fronts, internal waves, and isolated eddy 


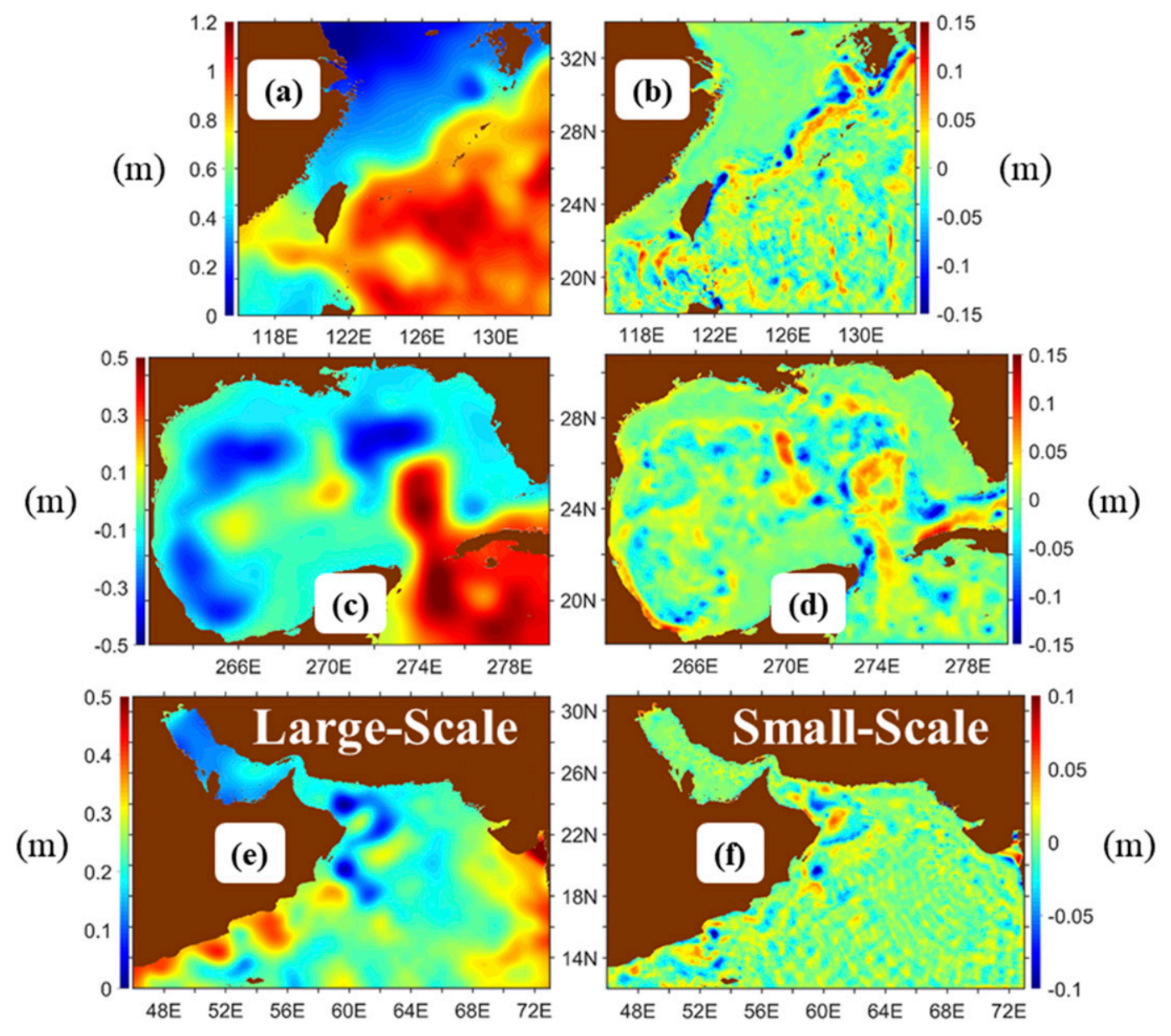

FIG. 4. Model (left) large-scale and (right) small-scale sea surface height (SSH; m) for (a),(b) the western Pacific, (c),(d) Gulf of Mexico, and (e),(f) Arabian Sea. See Eq. (1) for more details on how the two scales were decomposed.

features. Information averaged over the full domain may not lead to conclusions appropriate for just submesoscale eddies. Therefore, it is advantageous to isolate coherent submesoscale eddy features. The first step to identifying submesoscale eddies is by evaluating the Okubo-Weiss parameter (Okubo 1970; Weiss 1991) within the small-scale fields:

$$
\begin{aligned}
& W=S_{n}^{2}+S_{s}^{2}-\zeta^{2}, \\
& S_{n}=\partial_{x} u-\partial_{y} v ; \quad S_{s}=\partial_{x} v+\partial_{y} u ; \quad \zeta=\partial_{x} v-\partial_{y} u,
\end{aligned}
$$

where $S_{n}$ and $S_{s}$ are the normal and shear components of the strain and $\zeta$ is the relative vorticity. Computations use the small-scale surface velocity fields. Per Eq. (3), negative values of $W$ provide regions for which rotation dominates strain. Figure 5 provides a visualization of how the Okubo-Weiss mask identifies isolated vortices in the small-scale fields. We follow the methods outlined by Kurian et al. (2011). Surface small-scale velocities [obtained by Eq. (1)] were input to compute $W$ per Eq. (3). At each time step, the instantaneous fields of $W$ were normalized by the corresponding full field standard deviation. This normalized field is labeled $W_{N}$ for the remainder of the section. The normalized field $W_{N}$ was found to be noisy, so the $W_{N}$ field was filtered in order to accentuate coherent features. The same filter $F$ [Eq. (2)] was used, but the half-width was reduced to eight grid points $(\sim 8 \mathrm{~km})$. Closed contours of smoothed $W_{N}$ less than -0.2 were retained (Fig. $5 b$ ). The -0.2 contour threshold has been used in prior studies (Isern-Fontanet et al. 2003; Henson and Thomas 2008; Williams et al. 2011, and references therein). In addition to identifying eddies, the closed contours identify noneddy features such as filaments and squirts. A circularity test removed these unwanted features. The circularity of each closed contour, compared to a perfect circle occupying the same area, was tested and any feature with error greater 

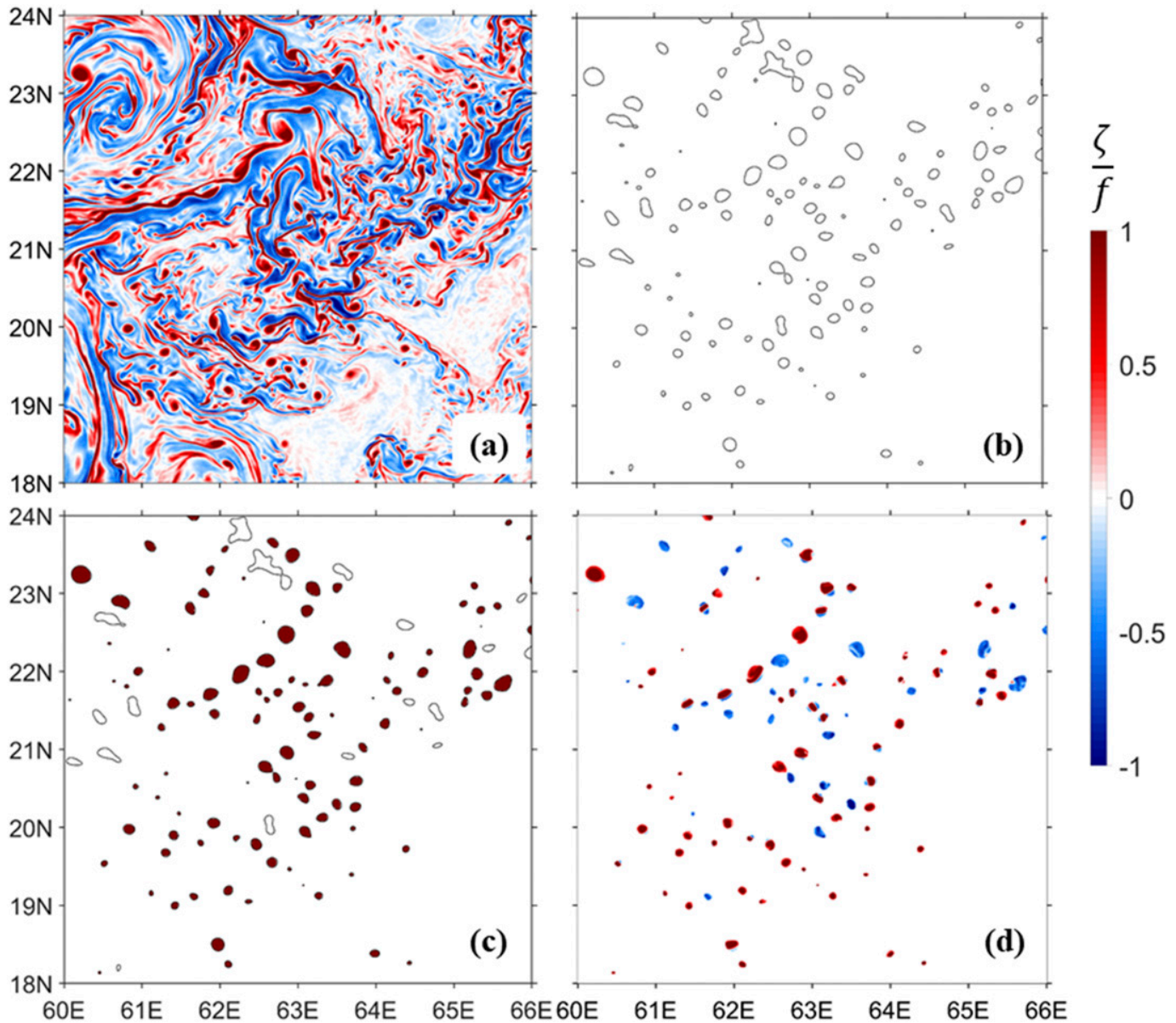

FIG. 5. Example of how the Okubo-Weiss mask was generated and applied. (a) Snapshot of small-scale surface vorticity in the Arabian Sea. The Okubo-Weiss parameter was calculated based on the instantaneous small-scale surface velocity field [Eq. (1)] and closed contours ( -0.2$)$ are shown in (b). (c) A circularity test was applied to each closed contour of (b) to retain eddying features. Retained features are highlighted in red, and discarded features are outlined and not filled. (d) The mask generated in (c) was applied to the small-scale surface vorticity in (a) to isolate the vorticity of the small-scale eddies. Submesoscale eddies were defined as those identified small-scale eddies with mean $|\zeta / f| \geq 1$ over the identified closed contour. All vorticities have been normalized by the Coriolis parameter.

than $35 \%$ was discarded (Kurian et al. 2011). The results of these criteria identify strong isolated vortices (Fig. 5c). Finally, we used the retained contours to isolate the features of interest. The results of applying the Okubo-Weiss mask to the surface small-scale vorticity field are shown in Fig. 5d.

An alternate eddy identification method was also tested (Hua and Klein 1998). The method is a generalization of the traditional Okubo-Weiss, taking into account the Lagrangian acceleration of the strain and vorticity fields that are potentially important components of the fast evolving submesoscale eddy dynamics. The method compared favorably with traditional Okubo-Weiss (results are not shown here). Both identified the same features, though each differed in magnitude of the criterion, an outcome anticipated by
Hua and Klein (1998). Therefore, we chose to use the more widely utilized traditional Okubo-Weiss method described above to identify the small-scale eddies in the model fields.

The spatial variability of the small-scale field contains significant energy at wavelengths of $50 \mathrm{~km}$ and longer (Fig. 3c), suggesting that some of the larger identified eddies could be "small mesoscale," a regime dynamically distinct from submesoscales. Therefore, we define submesoscale eddies as isolated small-scale vortices with mean $|\zeta / f|$, over the closed near-circular contours, greater than or equal to one (McWilliams 2016; D'Asaro et al. 2018). The submesoscale eddy selecting Okubo-Weiss mask is used in the subsequent analyses to examine variables such as temperature and salinity within the identified submesoscale 

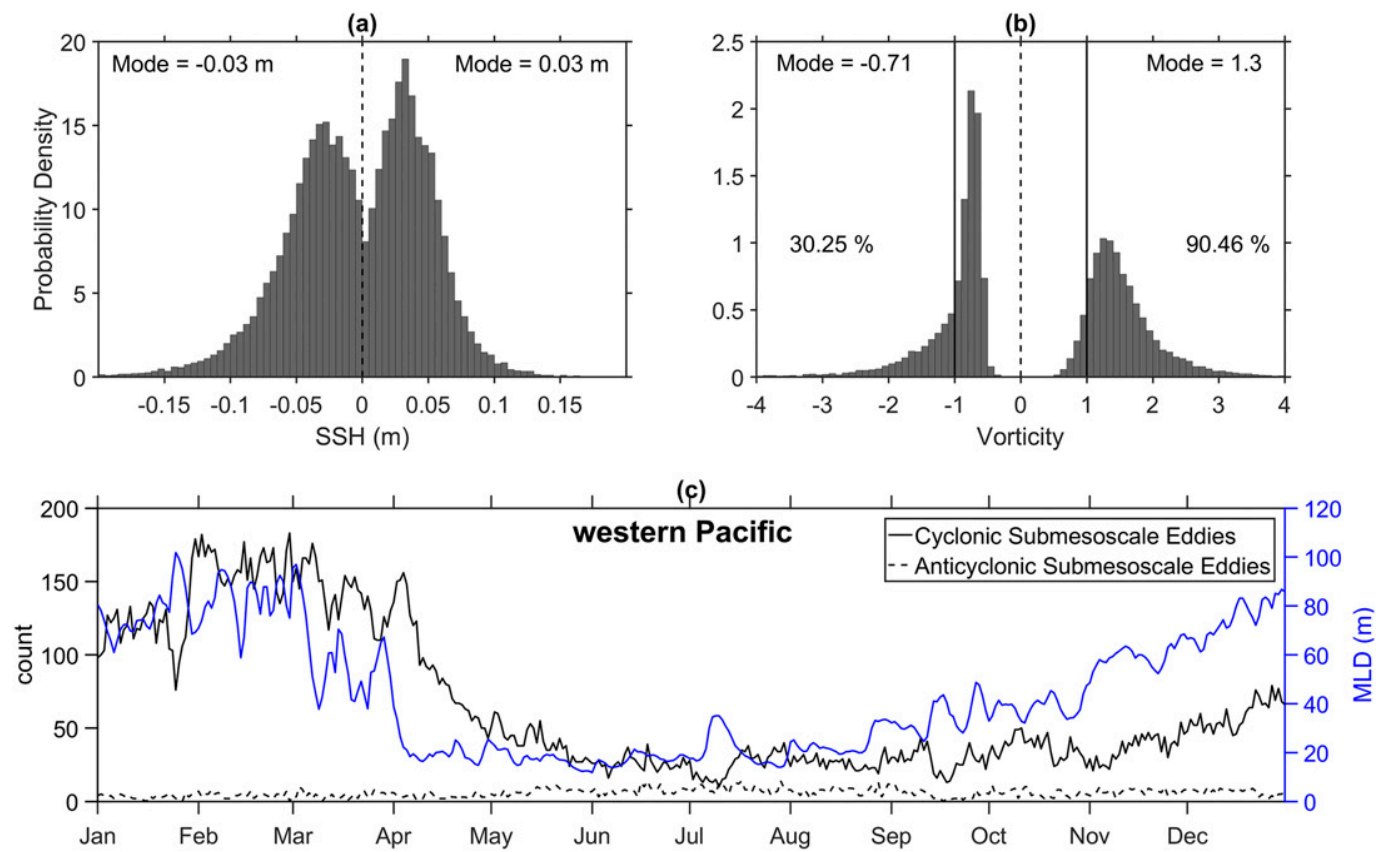

FIG. 6. Small-scale eddy statistics for the western Pacific. Small-scale eddies were selected based on the process described in Fig. 5. Small-scale cyclonic and anticyclonic eddy histograms of (a) SSH (m) and (b) surface vorticity normalized by the Coriolis parameter. In (b) the vertical black lines denote the $\zeta / f \pm 1$ criterion used to identify submesoscale eddies. (c) The number of submesoscale cyclonic (black line) and anticyclonic (dashed black line) eddies as well as the area-averaged MLD (m; blue line) at each 0000 UTC time step across the 2016 model integration period.

eddies at the surface and with depth. This allows dynamics and vertical relations to be characterized specifically with regard to the features defined here as submesoscale. As we show, the vertical structures within the submesoscale eddies are distinctly different from the large scale.

To summarize, we have defined the following:

Large-scale fields: A spatial filter with length scale $\sim 50 \mathrm{~km}$ (approximate Rossby radius of deformation) applied to the model fields provides the large scale.

Small-scale fields: The original model fields have the large-scale fields subtracted to provide the small scale.

Small-scale eddies: The Okubo-Weiss mask, calculated using the small-scale surface velocity, with smoothed $W_{N} \leq-0.2$ and passing a circularity test provides the small-scale eddies.

Submesoscale eddies: The small-scale eddies are segregated by identifying those with Rossby number averaged over the eddy closed contour having amplitude $\geq 1$.

Subsequent analyses identify which of these are used, with the majority focused on the submesoscale eddies.

\section{Results}

\section{a. Submesoscale eddy statistics}

We begin by analyzing submesoscale eddy statistics for each of the three regions under consideration. The analysis uses the small-scale surface velocity and SSH provided by Eq. (1) and isolated small-scale eddies provided by the Okubo-Weiss mask. Only grid points with at least $1000-\mathrm{m}$ water depth were considered. Cyclonic and anticyclonic small-scale eddies were identified by the sign of the surface vorticity averaged over the eddy closed contours. In the western Pacific (Fig. 6), small-scale eddy SSH has a bimodal distribution with peaks at $3 \mathrm{~cm}$ (anticyclonic) and $-3 \mathrm{~cm}$ (cyclonic) (Fig. 6a). The cyclonic distribution (negative SSH) has a much broader tail with a relatively large set of values having magnitudes $\geq 10 \mathrm{~cm}$. Regional smallscale eddy vorticity normalized by the Coriolis parameter has a bimodal distribution at peaks of 1.3 (cyclonic) and -0.71 (anticyclonic), with the former having a broader tail (Fig. 6b). This suggests that small-scale cyclonic eddies support a much wider range of higher magnitude vorticity than the small-scale anticyclonic eddies. This is consistent with theoretical, modeling, and observational work suggesting that strong anticyclonic 

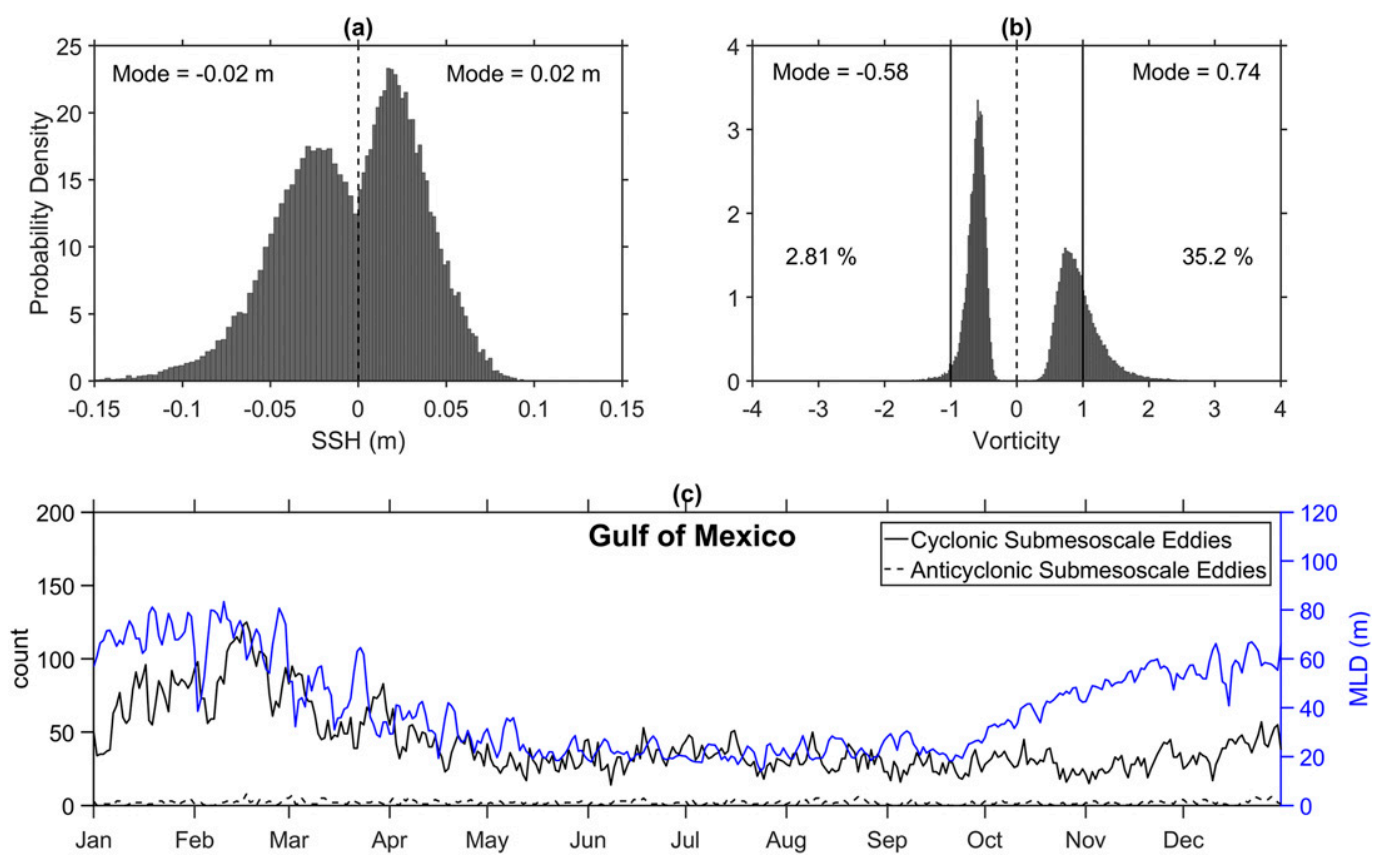

FIG. 7. Small-scale eddy statistics for the Gulf of Mexico. Small-scale eddies were selected based on the process described in Fig. 5. Small-scale cyclonic and anticyclonic eddy histograms of (a) SSH (m) and (b) surface vorticity normalized by the Coriolis parameter. In (b) the vertical black lines denote the $\zeta / f \pm 1$ criterion used to identify submesoscale eddies. (c) The number of submesoscale cyclonic (black line) and anticyclonic (dashed black line) eddies as well as the area-averaged MLD (m; blue line) at each 0000 UTC time step across the 2016 model integration period.

motions $(\zeta<-f)$ have more stringent stability criteria and therefore tend to be less numerous than their cyclonic counterparts (Hoskins and Bretherton 1972; Shcherbina et al. 2013). Vertical black lines mark $\zeta / f \pm 1$ in Fig. 6b, which is the limit of our criterion for classifying a submesoscale eddy. In the western Pacific, a majority of the cyclonic small-scale eddies can be classified as submesoscale $(90.46 \%)$, while only a minority $(30.25 \%)$ of the anticyclonic small-scale eddies can be classified as submesoscale. The number of cyclonic and anticyclonic submesoscale eddies are compared with the areaaveraged MLD (Fig. 6c). The MLD was calculated using a $0.2^{\circ} \mathrm{C}$ temperature criterion, which assumes that the isothermal layer is a good approximation of the isopycnal layer. Observational evidence suggests that this is reasonable for the three regions under investigation (Helber et al. 2012). Additionally, an analysis using our model results comparing the MLD calculated using the density and temperature criteria showed strong correspondence between the two estimates (results are not shown here). Figure $6 \mathrm{c}$ suggests a strong seasonal connection between the number of submesoscale eddies and the depth of the mixed layer. This finding is consistent with several past modeling and observational studies that have shown that submesoscale eddy motions dominate during the winter months when the mixed layer is deep and that internal wave dynamics dominate during the summer months when stratification is enhanced (Qiu et al. 2014; Callies et al. 2015; Rocha et al. 2016; Chang et al. 2018). Lagged correlation of several weeks occurs between the seasonal decrease in area-averaged MLD and the decrease in submesoscale eddy count between March and April.

In the Gulf of Mexico (Fig. 7), the cyclonic smallscale eddy SSH peak magnitude is $-2 \mathrm{~cm}$ (Fig. 7a). For the anticyclonic small-scale eddies, the peak SSH magnitude is $2 \mathrm{~cm}$. A bimodal peak in small-scale eddy vorticity is evident in the region (Fig. 7b), but the peaks are lower magnitude than the western Pacific: 0.74 and -0.58 for the cyclonic and anticyclonic smallscale eddies, respectively. In the Gulf of Mexico, only a minority of the small-scale eddies can be classified as submesoscale eddies: $35.2 \%$ for the cyclonic eddies and $2.81 \%$ for the anticyclonic eddies. Submesoscale eddy count is again strongly correlated with the areaaveraged MLD (Fig. 7c).

The aforementioned trends in small-scale eddy SSH (Fig. 8a) and vorticity (Fig. 8b) continue in the Arabian Sea, although a higher percentage of small-scale cyclonic 

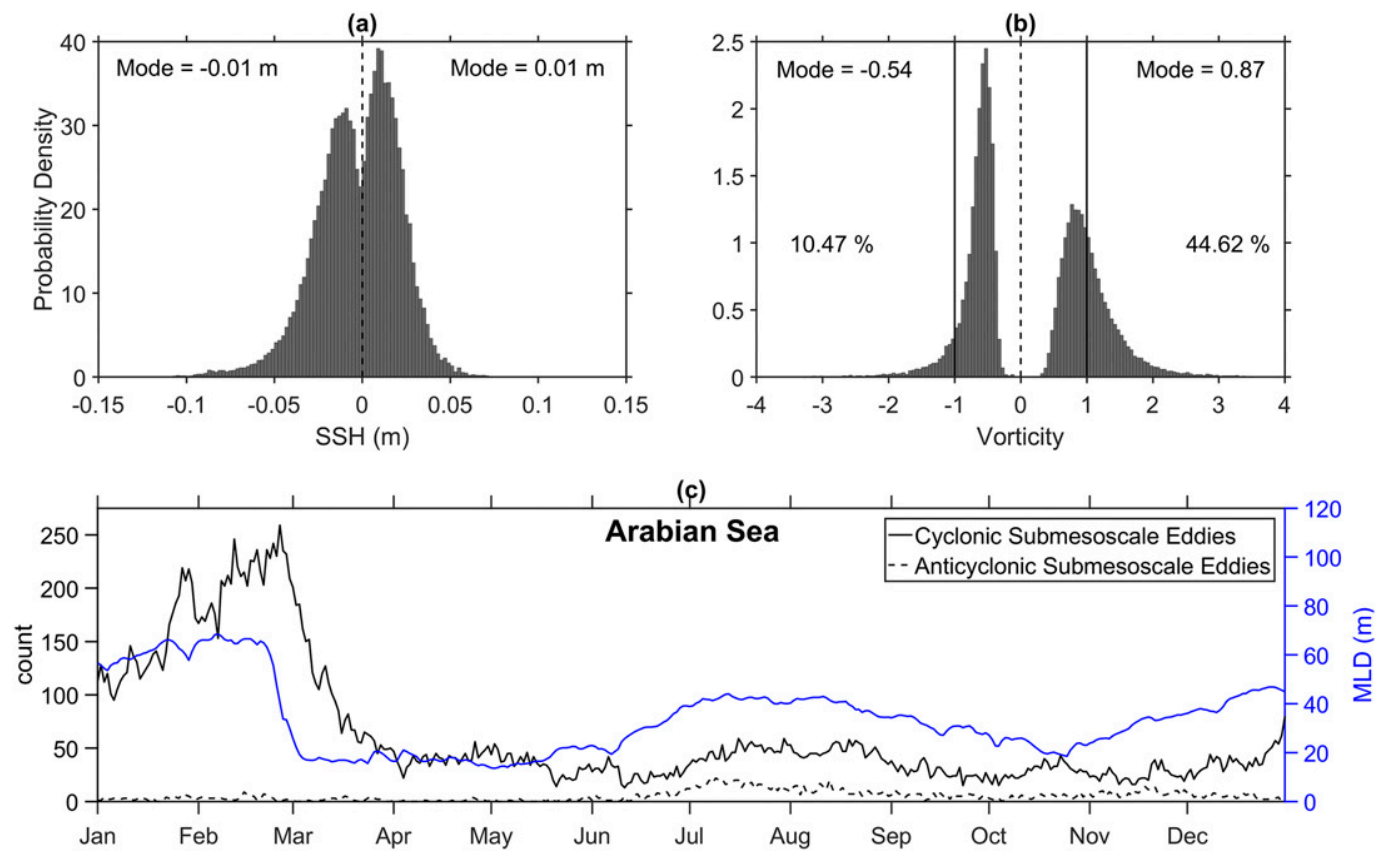

FIG. 8. Small-scale eddy statistics for the Arabian Sea. Small-scale eddies were selected based on the process described in Fig. 5. Small-scale cyclonic and anticyclonic eddy histograms of (a) SSH (m) and (b) surface vorticity normalized by the Coriolis parameter. In (b) the vertical black lines denote the $\zeta / f \pm 1$ criterion used to identify submesoscale eddies. (c) The number of submesoscale cyclonic (black line) and anticyclonic (dashed black line) eddies as well as the area-averaged MLD (m; blue line) at each 0000 UTC time step across the 2016 model integration period.

(44.62\%) and anticyclonic (10.47\%) eddies can be classified as submesoscale eddies than was observed in the Gulf of Mexico. In the Arabian Sea, the number of cyclonic eddies is substantially greater than the number of anticyclonic eddies with strong correlation with deep winter mixed layers (Fig. 8c), as was observed in the other two regions. In the Arabian Sea, the temporal lag between submesoscale eddy count and MLD is more prominent than in the western Pacific. The decline in MLD occurs at the beginning of March, with a strong drop in cyclonic eddy count not beginning for approximately a week. This suggests a cessation of strong submesoscale eddy creation as the mixed layer shallows, as well as a time period in which the already existing submesoscale eddies are dissipated. Additionally, a MLD maximum between July, August, and September also results in an increase in the number of submesoscale eddies. This increase in summer MLD can be attributed to the southwest monsoon season and provides additional evidence linking submesoscale eddy counts to the magnitude of the regional MLD.

Several trends are consistent across the three domains: 1) cyclonic small-scale eddy probability distributions have larger peak magnitudes for vorticity and longer tails for vorticity and SSH than the anticyclonic small-scale eddies, 2) the number of cyclonic submesoscale eddies is greater than the number of anticyclonic submesoscale eddies, and 3 ) the seasonal cycle in the number of submesoscale eddies correlates well with the depth of the area-averaged mixed layer. All three findings are consistent with prior studies using both models and observations. The latter most point requires a note of caution. The connection between the number of submesoscale eddies and the depth of the mixed layer is evident. However, the almost complete disappearance of submesoscale eddies during the summer months cannot be definitively confirmed by these results. As the mixed layer shallows in the summer, the mixed layer Rossby radius of deformation decreases. The horizontal length scales of submesoscale eddies are hypothesized to be linked to this quantity, just as the horizontal length scales of mesoscale eddies are linked directly to the first baroclinic Rossby radius of deformation (Thomas and Ferrari 2008; McWilliams 2016). Thus, in the summer the ocean may support smaller submesoscale eddies that are not resolved by the $1-\mathrm{km}$ simulations used in this study. The seasonal cycle of submesoscale eddy count will need to be more thoroughly investigated by a realistic, higherresolution model that resolves a much larger set of spatial scales. 
Submesoscale eddies have now been defined and classified. For the remainder of the paper, when "submesoscale" is referenced, it refers specifically to a smallscale eddy identified by Okubo-Weiss with mean $|\zeta / f| \geq 1$ over the identified closed contour.

\section{b. Submesoscale eddy vertical covariances}

A fundamental component of variational data assimilation is state variable covariance. Mesoscale covariances have been developed over decades in order to best assimilate large-scale nadir altimetry observations. Submesoscale eddy covariances, however, are poorly understood. This section provides a model-based approximation of these region specific depth-depth covariances for temperature, salinity, and specific volume anomalies (SVA; i.e., normalized density anomalies):

$$
\mathrm{SVA}=\frac{\rho_{o}\left(T_{o}, S_{o}, p\right)-\rho(T, S, p)}{\rho_{o}\left(T_{o}, S_{o}, p\right)},
$$

where $\rho_{o}$ is a reference density defined by $T_{o}=0^{\circ} \mathrm{C}$, $S_{o}=35 \mathrm{psu}$, and pressure $p$. To solve for submesoscale SVA, we calculated the unfiltered SVA and large-scale SVA and subtracted the latter from the former. The vertical covariance and correlation are defined by

$$
\begin{aligned}
C\left(z, z^{\prime}\right) & =\frac{(\mathbf{X}-\overline{\mathbf{X}})(\mathbf{X}-\overline{\mathbf{X}})^{\mathrm{T}}}{N-1}, \\
\boldsymbol{\sigma}_{z} & =\sqrt{\operatorname{diag}\left[C\left(z, z^{\prime}\right)\right]}, \quad \text { and } \\
\text { corr } & =\boldsymbol{\sigma}_{z}^{-1} C\left(z, z^{\prime}\right) \boldsymbol{\sigma}_{z}^{-1} .
\end{aligned}
$$

The term $\mathbf{X}$ is the model field arranged in a $L_{z} \times N$ matrix, where $L_{z}$ is the vertical dimension, $N=L_{x} L_{y} T$ is the matrix row length, $L_{x}$ and $L_{y}$ are the horizontal dimensions, and $T$ is the time dimension. The term $\overline{\mathbf{X}}$ is the row average taken along $N$. Finally, $\boldsymbol{\sigma}_{z}$ is the $L_{z} \times L_{z}$ diagonal matrix of the standard deviations in the vertical. Only grid points with at least 1000-m water depth were considered to focus on the open ocean dynamics. Using the definitions presented at the end of section $2 b(2)$, large-scale covariances use all data within the large-scale fields and submesoscale eddy covariances use only data within the identified submesoscale eddy closed contours (i.e., identified smallscale eddies with $|\zeta| f \mid \geq 1$ ). Large-scale and submesoscale eddy vertical covariances are presented and contrasted in the following paragraphs.

We begin by comparing the large-scale vertical temperature covariances to the corresponding submesoscale cyclonic and anticyclonic temperature covariances (Fig. 9). In each region, the large-scale temperature covariances have a primarily two-layer configuration. The first is within a near-surface layer, in which the temperature covariance is strongly positive between shallow depths $(<\sim 100 \mathrm{~m})$. In the western Pacific, this near-surface layer has weak negative covariance with depths below approximately $200 \mathrm{~m}$. A second, deeper layer of high magnitude positive covariance has strong off diagonal elements (i.e., depth layers covary over hundreds of meters). In general, the submesoscale eddy profiles have a much different vertical structure. In contrast with the general large-scale vertical temperature covariance structure, the submesoscale eddy profiles have weak covariance in the near-surface layer. Instead, the strongest temperature covariance begins below the near surface layer to approximately $200-\mathrm{m}$ depth. Some sign reversal is present in the anticyclonic profiles, but the magnitudes are much weaker than the strong positive covariance located primarily along the diagonal. Overall, mesoscale structures covary strongly within the upper and lower layers over hundreds of meters, and submesoscale structures covary over a more localized depth range just beneath the surface.

Salinity vertical covariances are shown in Fig. 10. Unlike with the temperature covariances, a general salinity vertical structure is not evident across the three regions. In the western Pacific, strong positive covariance in both the large-scale and submesoscale eddy profiles is confined to the very near surface environment. Pockets of negative covariance are evident, though their magnitudes are strongest in the large-scale profile. In the Gulf of Mexico, both the large-scale and submesoscale eddy profiles have strong positive covariance in the near-surface layer, but the large-scale profile has a secondary covariance maximum below $200 \mathrm{~m}$. The large-scale profile also has several pockets of higher magnitude negative covariance distributed over many depth levels. Some sign reversal is also evident in the submesoscale eddy covariance profiles, but the magnitudes are small compared with the strong positive covariance found primarily along the diagonal. Finally, in the Arabian Sea, the large-scale covariances are strongly positive in both the near-surface layer and subsurface extending beyond $500 \mathrm{~m}$. Unlike the other two regions, no sign reversal is observed within the top $500 \mathrm{~m}$ of the water column. Strong positive submesoscale eddy covariances are confined primarily within the nearsurface layer. Some weak off-diagonal negative elements are also apparent.

SVA combines temperature and salinity to provide density anomalies that control the stratification of the water column (Fig. 11). The large-scale SVA covariance vertical structure compares most closely to the temperature 

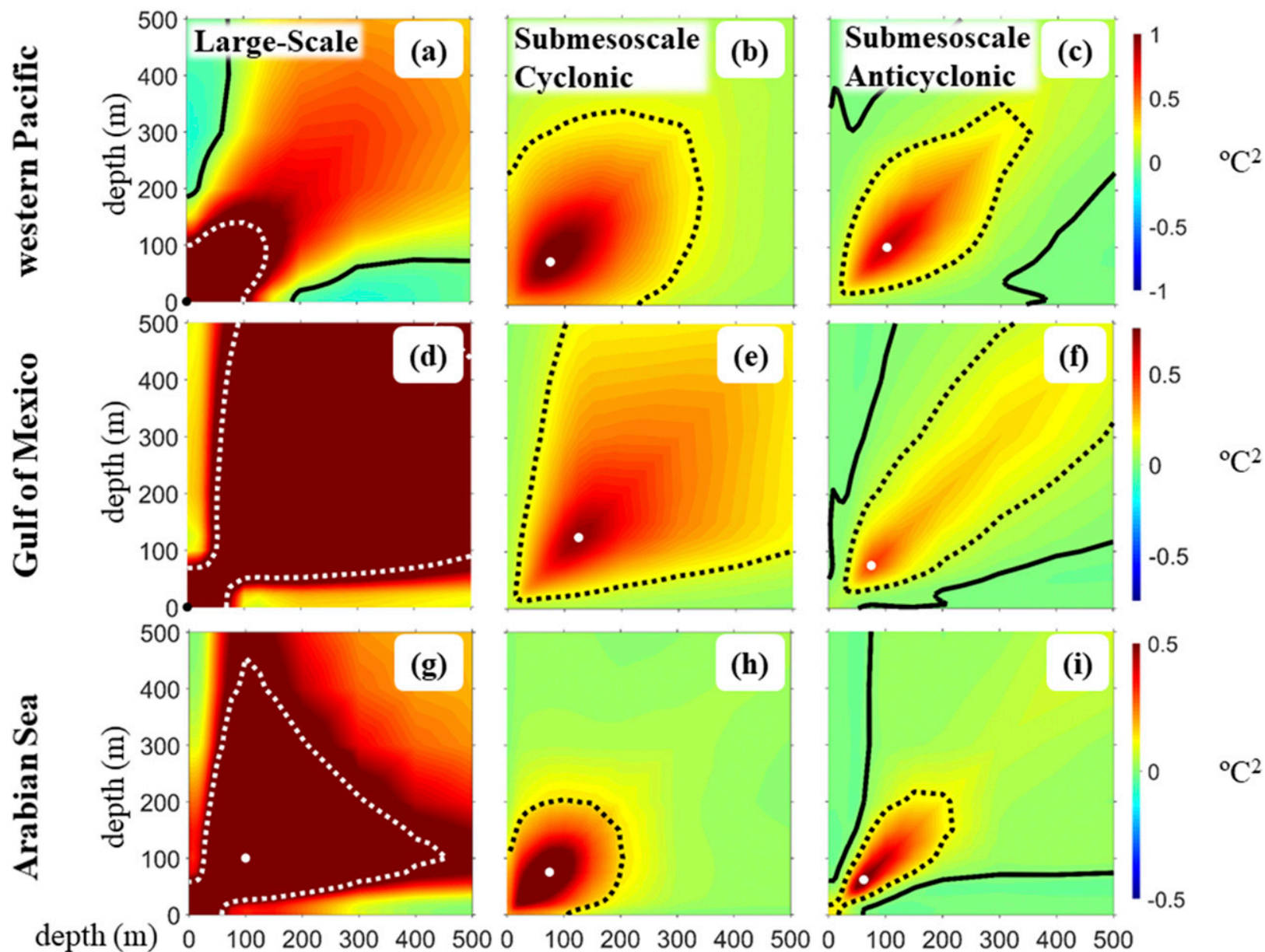

${ }^{\circ} \mathrm{C}^{2}$

FIG. 9. Vertical temperature-temperature covariances for (a)-(c) the western Pacific, (d)-(f) Gulf of Mexico, and (g)-(i) Arabian Sea. Color ranges are row specific. Solid lines denote the 0 covariance contour level. The solid dot denotes the maximum covariance value. Dashed lines enclose values $\geq 20 \%$ of the maximum covariance value. White and black lines do not denote different information but are used for visualization relative to the background color.

covariance structure (Fig. 9), with strong positive nearsurface covariance and a separate deeper layer of lower magnitude covariance extending beyond $500 \mathrm{~m}$. Within the deeper layer, strong off-diagonal elements are noted, suggesting strong correlation between vertical layers over hundreds of meters. Pockets of negative SVA covariance correspond primarily with negative temperature and salinity covariance in the western Pacific and Gulf of Mexico, respectively. The western Pacific submesoscale eddy profiles have strong positive covariance within the near-surface layer, a feature missing from the corresponding temperature covariances but present in the salinity covariances. Below this near surface layer, off diagonal elements are weak suggesting limited correlation between the vertical layers. Weak negative elements in the subsurface are evident, with greatest magnitude in the anticyclonic profile. In the Gulf of Mexico and Arabian Sea, weak submesoscale eddy covariances within the near-surface layer, observed in the corresponding temperature covariances, are noted. As with temperature, the strong covariances are found below the near surface layer and extend to a maximum depth of approximately $300 \mathrm{~m}$. Off diagonal elements are weak in each of the submesoscale eddy covariance profiles, suggesting weak correlation between vertical layers. In Gulf of Mexico, as in the western Pacific, weak negative covariance is located in the subsurface, with larger magnitudes found in the anticyclonic profile. In the Arabian Sea, negative elements are not observed in the top $500 \mathrm{~m}$ of the water column.

To summarize, the derived vertical covariances suggest that: 1) submesoscale eddy covariances are concentrated below the near surface layer to a maximum depth of approximately $300 \mathrm{~m}$ and 2) they have weak off diagonal elements, suggesting weak correlation between 

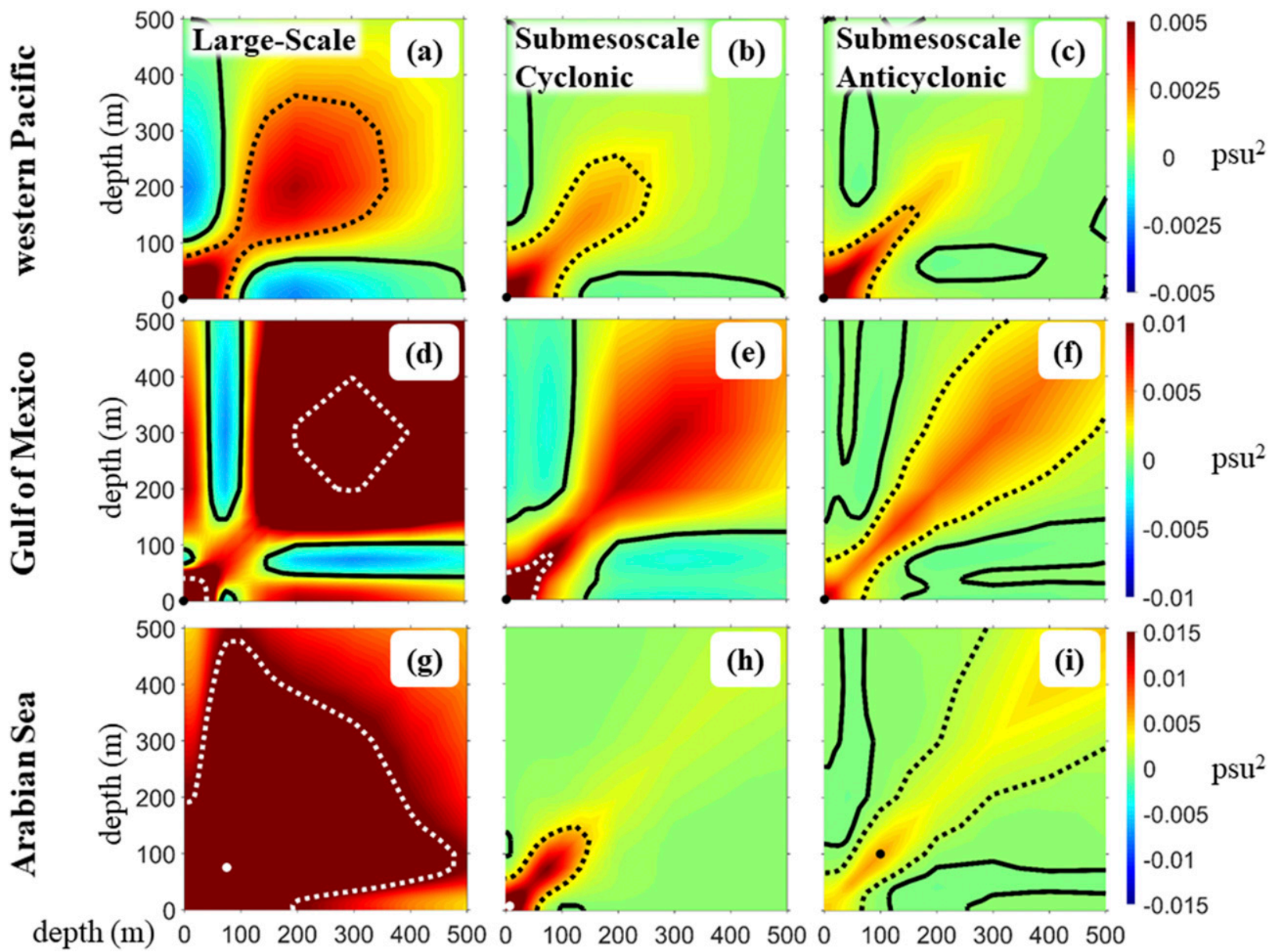

FIG. 10. Vertical salinity-salinity covariances for (a)-(c) the western Pacific, (d)-(f) Gulf of Mexico, and (g)-(i) Arabian Sea. Color ranges are row specific. Solid lines denote the 0 covariance contour level. The solid dot denotes the maximum covariance value. Dashed lines enclose values $\geq 20 \%$ of the maximum covariance value. White and black lines do not denote different information but are used for visualization relative to the background color.

vertical layers. This is in stark contrast to the large-scale covariances, which have a primarily two-layer configuration (near-surface layer and deeper layer) and have strong off diagonal elements, suggesting strong correlation within each of the two vertical layers. One caution should be noted. The covariance statistics are based on data across space and time, and the MLD varies across both. Thus, we cannot definitively state that the submesoscale eddy covariances are concentrated within or below the mixed layer. In the next section, we establish an alternative vertical grid that is a function of the MLD. This will allow us to estimate where the submesoscale eddy anomalies lie in the water column with respect specifically to the MLD.

\section{c. Submesoscale eddy vertical profiles}

In this section, we introduce an alternative vertical coordinate to better understand how the submesoscale eddy anomalies relate to the MLD. The term $z^{\prime}$ is the ratio of the true model depth to the MLD. We calculated mean small-scale vertical profiles on the $z^{\prime}$ grid within identified cyclonic and anticyclonic submesoscale eddies. These represent anomalies within submesoscale eddies from the large-scale background. Each profile in the mean was placed on the $z^{\prime}$ grid using the unfiltered MLD at the associated grid point within the identified submesoscale eddy. The results for temperature are shown in Fig. 12. In each of the three regions, anomalies from the large-scale background within the cyclonic submesoscale eddies are negative. The cyclonic submesoscale profiles are nearly constant throughout the mixed layer, steadily increase in magnitude below the base of the mixed layer, and reach peak magnitude at $z^{\prime}$ $\sim 2$ (i.e., 2 times the MLD). The exception being the Gulf of Mexico which features a slightly deeper $z^{\prime}$ maximum. The anticyclonic submesoscale profiles are 


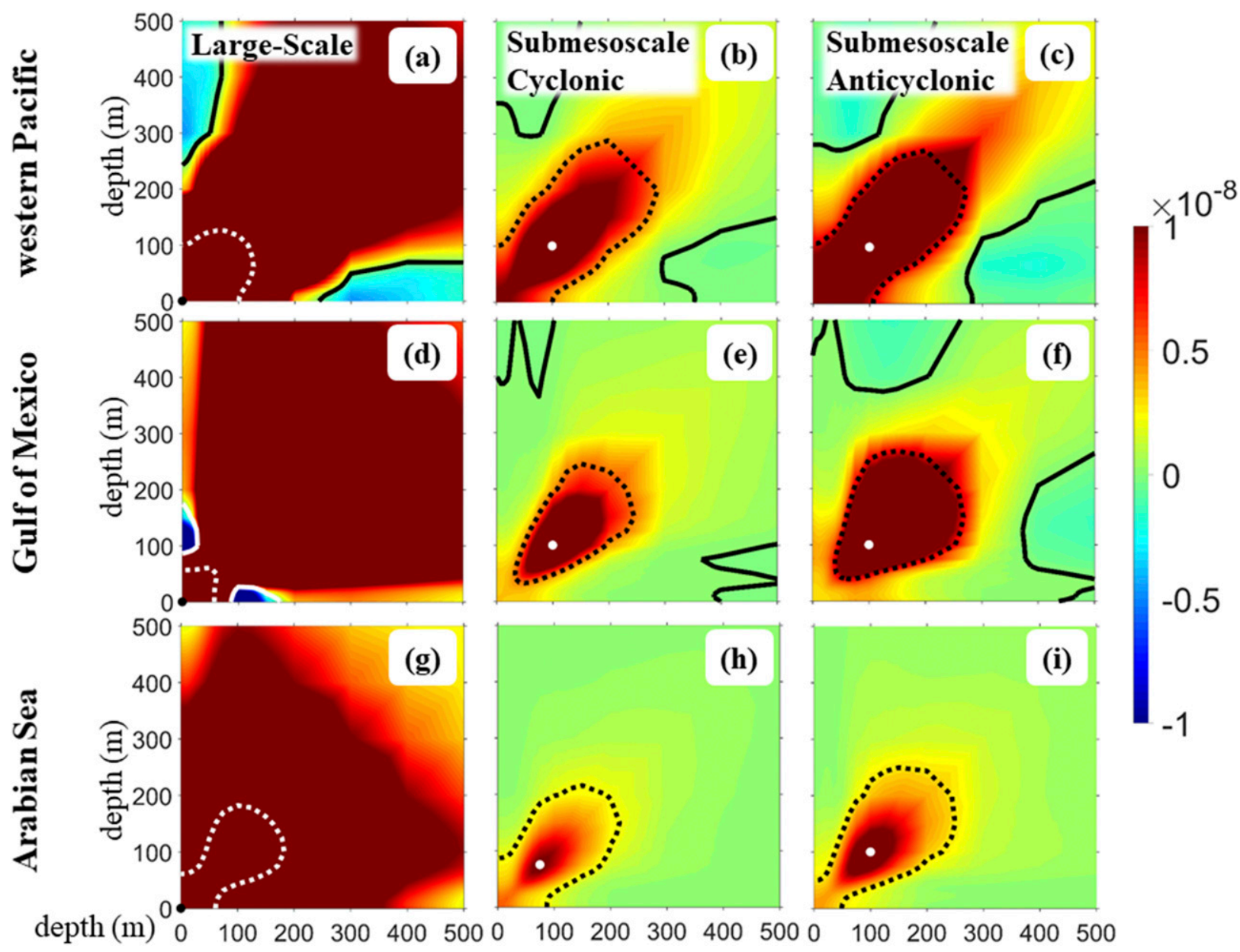

FIG. 11. Vertical SVA-SVA (specific volume anomalies) covariances for (a)-(c) the western Pacific, (d)-(f) Gulf of Mexico, and (g)-(i) Arabian Sea. Solid lines denote the 0 covariance contour level. The solid dot denotes the maximum covariance value. Dashed lines enclose values $\geq 20 \%$ of the maximum covariance value. White and black lines do not denote different information but are used for visualization relative to the background color.

near zero at the surface and increase in magnitude throughout the mixed layer until reaching peak magnitude at $z^{\prime} \sim 1$ (i.e., the base of the mixed layer). Despite differences in overall magnitude, the vertical structure is similar in all three regions. This corresponds well with the temperature covariances detailed in the prior section. We observed weak covariances in the very near surface, and larger magnitudes between 100 and $300 \mathrm{~m}$ (Fig. 9). Although in the analysis of section $3 b$ we could not determine how the peak covariances related to the MLD, Fig. 12 confirms that the submesoscale cyclonic and anticyclonic temperature anomalies are concentrated within the proximity of the base of the mixed layer.

The submesoscale mean salinity profiles (Fig. 13) are more complicated and show a less coherent structure when compared with the mean temperature profiles. For the cyclonic submesoscale eddies, salinity anomalies are positive and near constant throughout the mixed layer and then begin a reversal of sign below. The anticyclonic submesoscale profiles reverse twice within the water column with the first local maximum anomaly corresponding with the base of the mixed layer. Temperature and salinity both influence the mean SVA profiles (Fig. 14). Both the cyclonic and anticyclonic submesoscale SVA profiles resemble the temperature profiles closely. For the cyclonic submesoscale profile, negative anomalies are near constant within the mixed layer, become increasingly negative starting at the MLD, and then reach peak magnitude at approximately 2 times the MLD (peak $z^{\prime}=2.3,2.2$, and 1.9 for the western Pacific, Gulf of Mexico, and Arabian Sea, respectively). For the anticyclonic submesoscale SVA profiles, anomalies begin near zero at the surface, become increasingly positive throughout the mixed layer, and then reach peak magnitude just below the MLD (peak $z^{\prime}=1.1,1$, 


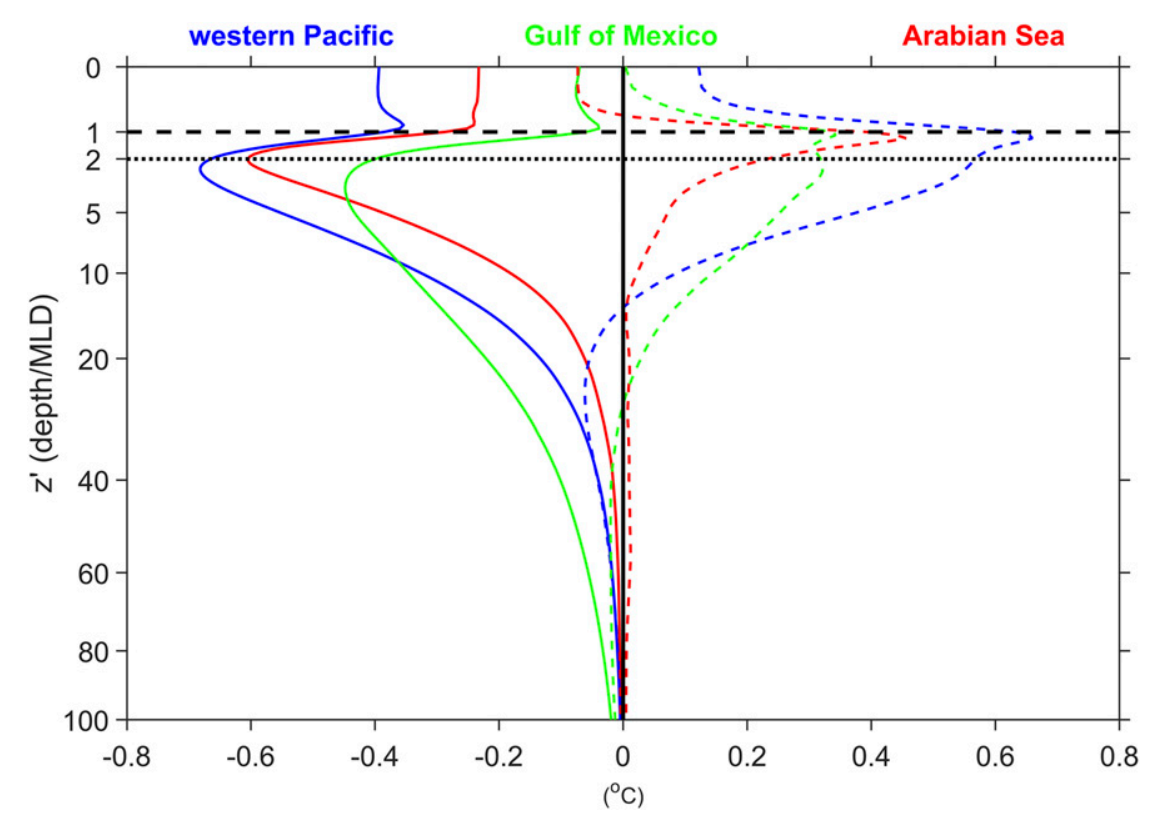

FIG. 12. Mean vertical distribution of small-scale temperature $\left({ }^{\circ} \mathrm{C}\right)$ within submesoscale cyclonic (solid line) and anticyclonic (dashed line) eddies in the western Pacific (blue lines), Gulf of Mexico (green lines), and Arabian Sea (red lines). The $z^{\prime}$ is an alternative vertical coordinate defined as the ratio of the model depth to the MLD. Each profile in the mean was placed on the $z^{\prime}$ grid using the unfiltered MLD at the associated grid point within the identified submesoscale eddy. Dashed, horizontal lines demark $z^{\prime}$ values of 1 and 2.

and 1.2 for the western Pacific, Gulf of Mexico, and Arabian Sea, respectively).

The illustration described in section 1, featuring a cyclonic submesoscale eddy propagating around an anticyclonic mesoscale eddy (Fig. 1), corresponds well with the statistics shown in this section. The black dot in Fig. 1d shows the depth twice the MLD within the cyclonic submesoscale eddy. At this depth, we observe significant small-scale density anomalies. While the large-scale isopycnals are displaced downward beyond $500 \mathrm{~m}$, the small-scale density anomalies caused by the cyclonic submesoscale eddy extend to only $300 \mathrm{~m}$. Thus, the results suggest a connection between submesoscale eddies and the mixed layer, and in addition suggest that they have significant baroclinic effects beyond the mixed layer. Recent observational evidence supports these model-based results by showing strong submesoscale cyclonic vorticity extending up to $200 \mathrm{~m}$ beyond the base of the mixed layer (Yu et al. 2019).

The vertical structure of the mean temperature anomalies within the submesoscale eddies indicates some interesting properties. The weak temperature anomalies within the mixed layer suggest that pressure anomalies due to SSH within submesoscale eddies are not compensated by density variations within the mixed layer. Instead, the submesoscale eddies have relatively uniform structure throughout the mixed layer.
Beneath the mixed layer, temperature primarily controls the density anomalies, and the temperature anomalies gradually reduce the horizontal pressure gradient associated with the SSH. This is consistent with a steady state inviscid thermal wind balance. The implication is that submesoscale eddy velocity is relatively uniform through the mixed layer and decreases with depth. The salinity anomalies indicate both positive and negative variations within submesoscale eddies. The salinity variations are consistent with upward vertical displacement of the larger-scale isohalines in the opposite direction of the downward SSH displacement.

Vertical velocity may also play a role in the observed vertical displacements. Past work has indicated that submesoscale features induce strong vertical advection (Klein and Lapeyre 2009; Koszalka et al. 2009; Zhong and Bracco 2013; Lévy et al. 2012; Brannigan 2016; Zhong et al. 2017; Su et al. 2018; Lévy et al. 2018). There are two sources of vertical velocity. The submesoscale eddy horizontal velocity decreases with depth, which implies vertical shear and thus Ekman transport, horizontal divergence, and ultimately vertical velocity within submesoscale eddies. Vertical velocity due to the submesoscale eddy vertical shear would be expected to be symmetrically centered on circular symmetric eddies. The second source of vertical velocity is the straining of horizontal buoyancy gradients leading 


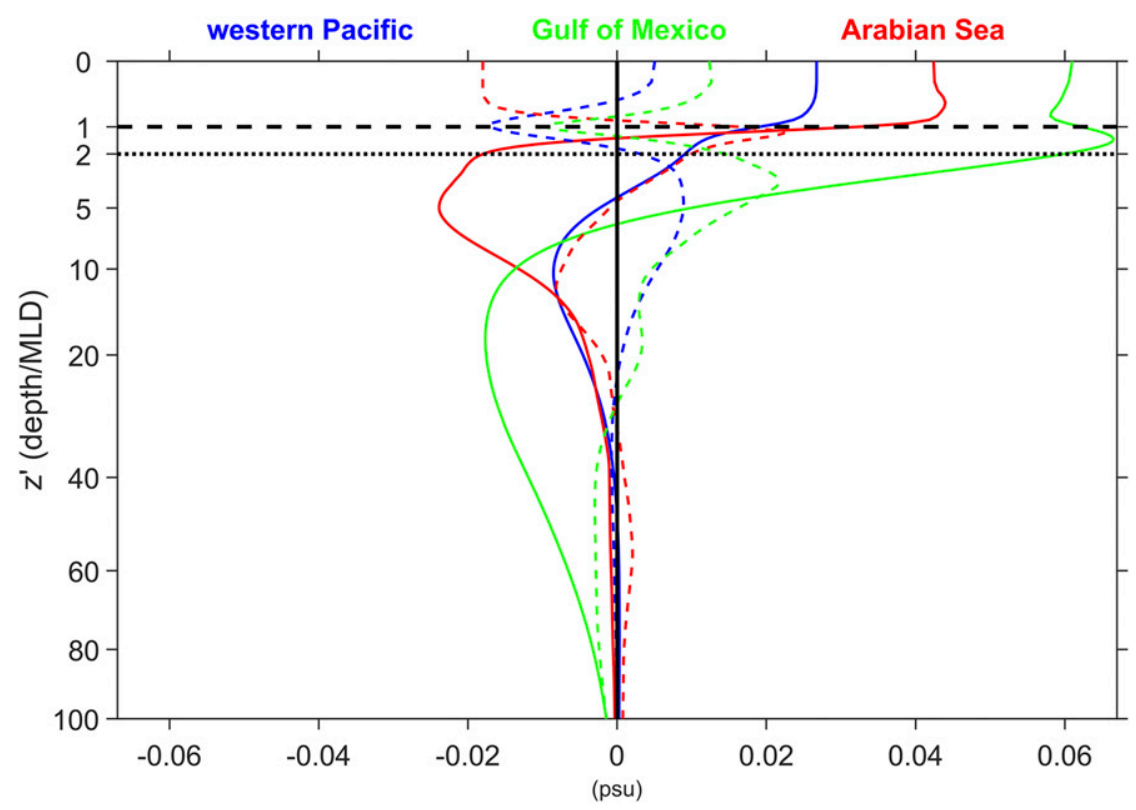

FIG. 13. Mean vertical distribution of small-scale salinity (psu) within submesoscale cyclonic (solid line) and anticyclonic (dashed line) eddies in the western Pacific (blue lines), Gulf of Mexico (green lines), and Arabian Sea (red lines). The $z^{\prime}$ is an alternative vertical coordinate defined as the ratio of the model depth to the MLD. Each profile in the mean was placed on the $z^{\prime}$ grid using the unfiltered MLD at the associated grid point within the identified submesoscale eddy. Dashed, horizontal lines demark $z^{\prime}$ values of 1 and 2 .

to frontogenesis (Hoskins and Bretherton 1972; Hoskins 1982). Because the straining occurs along the fronts of eddies and depends on the buoyancy distribution, the vertical velocity is not necessarily symmetrically centered on the eddy core. Examination of the vertical velocity computed from integrating horizontal divergence from the model free surface downward indicates a mixture of these phenomena (results are not shown here). The straining of buoyancy is large around submesoscale features because of the small horizontal scales, and frontogenesis-driven vertical velocity is prevalent throughout the model results. The salinity anomalies within the submesoscale eddies are consistent with isopycnal displacements due to surface pressure anomalies and reduced pressure with depth. There is not clear indication that the vertical velocity is producing the submesoscale salinity structure we have documented in this section.

Thus, the model-derived vertical structures are likely produced by a combination of the pressure compensation and vertical velocity phenomena. These mechanisms should be explored more rigorously in future work. Our algorithm identifying circular submesoscale features preferentially selects mature submesoscale eddies with clearly closed circulations. An analysis of the subsurface evolution of submesoscale eddies would more confidently elucidate the sequence of events that lead to the vertical structures we have documented here. Idealized experiments exist (e.g., Brannigan et al. 2017; Morvan et al. 2019), but their focus was not on the setup of the submesoscale eddy associated subsurface temperature/salinity/density structure. Thus, realistic simulation and direct observation of these processes remain frontiers.

\section{d. Submesoscale eddy dynamics}

\section{1) DYNAMICAL CONSTRAINT}

In sections $3 b$ and $3 c$, we have demonstrated a clear difference between the large-scale and submesoscale eddy vertical structures. The results suggest that the submesoscale eddy features we have identified are governed by a set of dynamics unique from the wellstudied mesoscale phenomenon. Therefore, in this section, we test a series of dynamical balances that sequentially add terms from the Eulerian primitive equations. Our overarching interest is the interpretation of the SWOT observations, which are SSH. Therefore, we consider a surface dynamical relation associated specifically with surface elevation $\eta$ and surface velocity u. In this context, the three-dimensional equations of motion are applied in two dimensions just at the surface. Assuming frictionless motion on an $f$ plane in two dimensions, we begin with the following constraint: 


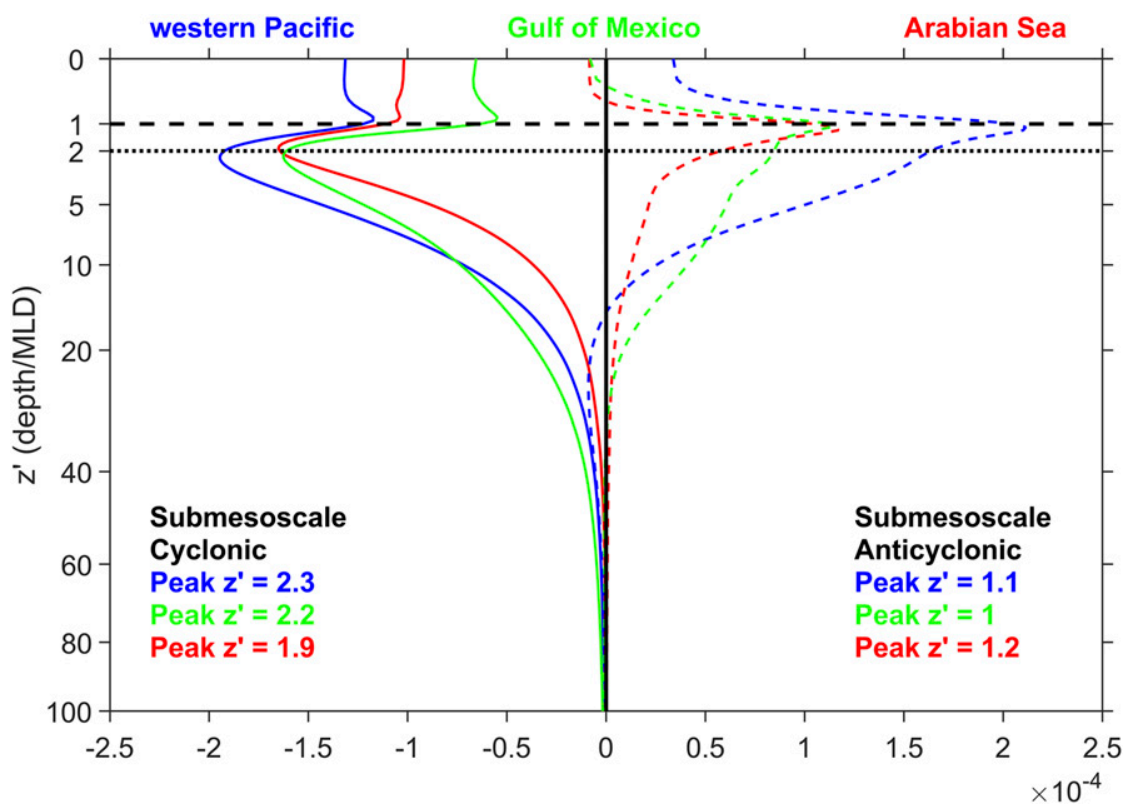

FIG. 14. Mean vertical distribution of small-scale specific volume anomalies (SVA) within submesoscale cyclonic (solid line) and anticyclonic (dashed line) eddies in the western Pacific (blue lines), Gulf of Mexico (green lines), and Arabian Sea (red lines). The $z^{\prime}$ is an alternative vertical coordinate defined as the ratio of the model depth to the MLD. Each profile in the mean was placed on the $z^{\prime}$ grid using the unfiltered MLD at the associated grid point within the identified submesoscale eddy. Dashed, horizontal lines demark $z^{\prime}$ values of 1 and 2 .

$$
\partial_{t} \mathbf{u}+(\mathbf{u} \cdot \nabla) \mathbf{u}+f \hat{\mathbf{k}} \times \mathbf{u}=-g \nabla \eta,
$$

where $f$ is the Coriolis parameter, $\hat{\mathbf{k}}$ is the vertical unit vector, and $g$ is gravitational acceleration. We begin the development of the submesoscale constraint by first decomposing the velocity vector and surface elevation into large- and small-scale components per Eq. (1):

$$
\begin{gathered}
\mathbf{u}=\mathbf{u}_{L}+\mathbf{u}_{S}, \\
\eta=\eta_{L}+\eta_{S} .
\end{gathered}
$$

This partition generates an expanded version of Eq. (9) that is a function of both large- and small-scale velocity and surface elevation. For the large-scale component, we assume that those motions are predominantly governed by

$$
\partial_{t} \mathbf{u}_{L}+\left(\mathbf{u}_{L} \cdot \nabla\right) \mathbf{u}_{L}+f \hat{\mathbf{k}} \times \mathbf{u}_{L}=-g \nabla \eta_{L} .
$$

Removing Eq. (12) from the expanded version of Eq. (9) yields

$$
\begin{aligned}
& \partial_{t} \mathbf{u}_{S}+\left[\left(\mathbf{u}_{L}+\mathbf{u}_{S}\right) \cdot \nabla\right] \mathbf{u}_{S}+\left(\mathbf{u}_{S} \cdot \nabla\right) \mathbf{u}_{L}+f \hat{\mathbf{k}} \times \mathbf{u}_{S} \\
& \quad=-g \nabla \eta_{S} .
\end{aligned}
$$

The terms are the tendency of the small-scale field, the advection of small-scale momentum by both the large and small scales, the advection of the large scales by the small scales, the Coriolis force due to the small-scale motions, and the surface pressure gradient at small scales. Assuming that the characteristic large length scales are much larger than the characteristic small length scales $L_{L} \gg L_{S}$, and that the velocities of the large and small scales are similar, the advection of the large scale by the small scale becomes small and yields:

$$
\partial_{t} \mathbf{u}_{S}+\left[\left(\mathbf{u}_{L}+\mathbf{u}_{S}\right) \cdot \nabla\right] \mathbf{u}_{S}+f \hat{\mathbf{k}} \times \mathbf{u}_{S}=-g \nabla \eta_{S} .
$$

Equation (14) agrees with our current understanding of Rossby number $O(1)$ submesoscale motions: 1) Eulerian time scales are relatively short $O(1)$ day, 2) larger-scale synoptic and mesoscale motions advect the submesoscale eddies, and 3) submesoscale eddies remain subject to the influence of planetary vorticity (Capet et al. 2008b; McWilliams 2016). Equation (14) is insufficient for dealing with internal wave motions and frontal physics that are present in our small-scale fields (Fig. 4). Limiting our dynamical relation to two dimensions in the horizontal forces us to neglect vertical stratification, therefore precluding the correct representation of wave dynamics (Lynett and Liu 2002). Because internal waves generate a small-scale $\mathrm{SSH}$, analysis of the surface submesoscale eddy fields using Eq. (14) will lead to errors due to internal waves. Our experimentation will 


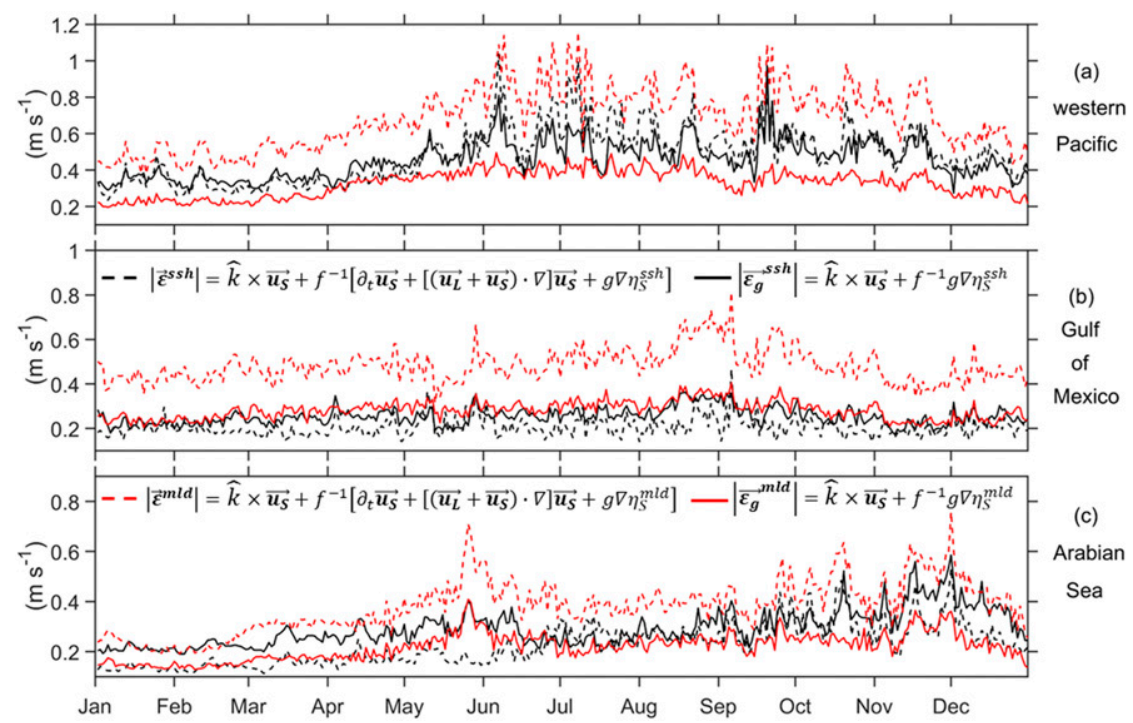

FIG. 15. Surface submesoscale eddy velocity error magnitudes for (a) the western Pacific, (b) Gulf of Mexico, and (c) Arabian Sea. The black lines used small-scale SSH for surface elevation, while the red lines used small-scale SVA integrated through the area-averaged MLD for surface elevation. Solid lines used only the Coriolis and surface elevation term [Eq. (17)], while the dashed lines used the tendency, advection, Coriolis, and surface elevation terms [Eq. (15)].

help illuminate how significantly the wave dynamics reduce the utility of this dynamical constraint on the submesoscale eddy field.

\section{2) ERror evaluation}

Equation (14) is expressed as functions of the largeand small-scale fields, and we use the filtered large- and small-scale fields as defined at the end of section $2 b(2)$. In this section, we evaluate errors with respect to Eq. (14) only within identified submesoscale eddies. Only submesoscale eddies located in regions of at least $1000-m$ water depth were considered. Finally, only 0000 UTC time steps were considered to limit the number of times individual submesoscale eddies were included in our statistics.

Errors are defined by

$$
\begin{aligned}
\boldsymbol{\epsilon} & =\hat{\mathbf{k}} \times \mathbf{u}_{S}+f^{-1}\left\{\partial_{t} \mathbf{u}_{S}+\left[\left(\mathbf{u}_{L}+\mathbf{u}_{S}\right) \cdot \nabla\right] \mathbf{u}_{S}+g \nabla \eta_{S}\right\} \\
|\boldsymbol{\epsilon}| & =\sqrt{\epsilon_{x}^{2}+\epsilon_{y}^{2}}
\end{aligned}
$$

where $|\boldsymbol{\epsilon}|$ is the magnitude of the error. Equation (16) was evaluated for each full field and then the submesoscale eddy selecting Okubo-Weiss mask was applied to isolate error magnitudes within the identified submesoscale eddies. The results presented in Fig. 15 are the errors averaged only over the identified submesoscale eddies for each time step. We begin by assuming that the submesoscale eddy surface elevation $\eta_{S}$ is best represented by the small-scale SSH. The solid black lines in Fig. 15 are the averaged errors when Eq. (15) is reduced to the geostrophic approximation:

$$
\boldsymbol{\epsilon}_{g}=\hat{k} \times \mathbf{u}_{S}+f^{-1} g \nabla \eta_{S} .
$$

Time-averaged error statistics for each region are summarized in Table 2. In the western Pacific (Fig. 15a), submesoscale eddy geostrophic errors have a temporal mean of $46 \mathrm{~cm} \mathrm{~s}^{-1}$ and errors have a seasonal maximum in boreal summer. This is consistent with the seasonal transition from a dominance of submesoscale eddies in winter to internal wave dominance in the summer (Qiu et al. 2014; Callies et al. 2015; Rocha et al. 2016; Chang

TABLE 2. Summary of submesoscale eddy errors $\left(\mathrm{cm} \mathrm{s}^{-1}\right)$. Angle brackets represent the time averaging of the error time series shown in Fig. 15. The error minimum for each region is bolded.

\begin{tabular}{cccc}
\hline \hline & $\begin{array}{c}\text { Western } \\
\text { Pacific }\end{array}$ & $\begin{array}{c}\text { Gulf of } \\
\text { Mexico }\end{array}$ & $\begin{array}{c}\text { Arabian } \\
\text { Sea }\end{array}$ \\
\hline$\left\langle\left|\boldsymbol{\epsilon}_{g}^{\text {ssh }}\right|=\hat{\mathbf{k}} \times \mathbf{u}_{S}+f^{-1} g \nabla \eta_{S}^{\text {ssh }}\right\rangle$ & 46 & 25 & 29 \\
$\left\langle\left|\boldsymbol{\epsilon}^{\text {ssh }}\right|=\hat{\mathbf{k}} \times \mathbf{u}_{S}+f^{-1}\left\{\partial_{t} \mathbf{u}_{S}\right.\right.$ & 48 & $\mathbf{2 0}$ & 23 \\
$\left.\left.\quad+\left[\left(\mathbf{u}_{m}+\mathbf{u}_{S}\right) \cdot \nabla\right] \mathbf{u}_{S}+g \nabla \eta_{S}^{\text {ssh }}\right\}\right\rangle$ & & & \\
$\left\langle\left|\boldsymbol{\epsilon}_{g}^{\text {mld }}\right|=\hat{\mathbf{k}} \times \mathbf{u}_{S}+f^{-1} g \nabla \eta_{S}^{\text {mld }}\right\rangle$ & $\mathbf{3 3}$ & 28 & $\mathbf{2 2}$ \\
$\left\langle\left|\boldsymbol{\epsilon}^{\text {mld }}\right|=\hat{\mathbf{k}} \times \mathbf{u}_{S}+f^{-1}\left\{\partial_{t} \mathbf{u}_{S}\right.\right.$ & 68 & 48 & 38 \\
$\left.\left.\quad+\left[\left(\mathbf{u}_{m}+\mathbf{u}_{S}\right) \cdot \nabla\right] \mathbf{u}_{S}+g \nabla \eta_{S}^{\text {mld }}\right\}\right\rangle$ & & & \\
\hline
\end{tabular}


et al. 2018). In the Gulf of Mexico, time averaged error magnitudes are much lower $\left(25 \mathrm{~cm} \mathrm{~s}^{-1}\right)$ and a strong seasonal cycle in error is absent. Again, the lack of strong barotropic tides in the Gulf of Mexico tends to generate weaker baroclinic tides, which inhibits the magnitude of internal wave contributions to SSH. Finally, in the Arabian Sea (Fig. 15c), mean error magnitude through time is $29 \mathrm{~cm} \mathrm{~s}^{-1}$.

For the Gulf of Mexico and Arabian Sea, expanding to a more complete set of Eulerian force balance terms [Eq. (15)] reduces error magnitudes across a majority of the time series (dashed black lines in Fig. 15). The timeaveraged error reductions from assuming only geostrophy to using the full set of terms are 5 and $6 \mathrm{~cm} \mathrm{~s}^{-1}$ for the Gulf of Mexico and Arabian Sea, respectively. These values correspond with percent decreases of $20 \%$ and $21 \%$. This finding is consistent with our hypothesis, whereby submesoscale currents have shorter Eulerian time scales (significant velocity tendency) and are forced by strong background currents (significant advection). In the western Pacific, however, time-averaged errors are slightly higher when using the full set of Eulerian terms $\left(2 \mathrm{~cm} \mathrm{~s}^{-1}\right)$. Although, the percent increase is much smaller (4\%) than the percent decreases observed in the Gulf of Mexico and Arabian Sea ( 20\%). Additionally, during the first 91 days of the year (January-March), using the full set of Eulerian terms produces slightly less error ( $35 \mathrm{vs} 32 \mathrm{~cm} \mathrm{~s}^{-1}$ ), suggesting a seasonal transition in higher errors when using Eq. (15) in the western Pacific, likely due to the increase in strong baroclinic tides in boreal summer. This internal wave influence on the submesoscale eddy momentum budget is explored more extensively later in this section. Finally, Eq. (15) with and without each of the advective terms was evaluated. In the Arabian Sea, for example, the second most accurate solution included small-scale advection and small-scale tendency and excluded large-scale advection, with only the solution including all of the terms producing lower errors. Including the largescale advection and small-scale tendency, and excluding small-scale advection, produced a solution comparable to geostrophy. These findings are consistent with a gradient wind balance approximation often cited for motions with Rossby number $O(1)$ (Capet et al. 2008b).

There is a prevailing hypothesis that the submesoscale eddy phenomenon is primarily confined within the mixed layer (e.g., Thompson and Lazar 2016; Bachman et al. 2017). We further test this hypothesis here by replacing surface elevation $\eta_{S}$ in Eqs. (15) and (17) with mixed layer integrated small-scale steric height instead of small-scale SSH. Small-scale steric height was calculated by

$$
\mathrm{SH}_{S}=\int_{0}^{z} \mathrm{SVA}_{S} d z
$$

where $\mathrm{SH}_{S}$ is the small-scale steric height, $z$ is a depth of integration, and $\mathrm{SVA}_{S}$ is the small-scale SVA [Eq. (5)]. For mixed layer integrated small-scale steric height $\mathrm{SH}_{S}^{\overline{\mathrm{MLD}}}$, Eq. (18) is evaluated by integrating through the mixed layer, which was taken to be an average over the domain at each respective time step. The reason for using an area average is that the MLD is strongly affected by frontogenesis (Jacobs et al. 2014), which produces filaments of shallow MLD mainly in fronts associated with mesoscale and submesoscale eddies. The dynamics supporting the submesoscale features are thought to be pressure gradients within the mixed layer, averaged within the eddies. We repeat the analysis of the prior paragraph using $\mathrm{SH}_{S}^{\overline{\mathrm{MLD}}}$ for $\eta_{S}$. The solid red lines of Fig. 15 are the averaged errors when assuming the geostrophic approximation [Eq. (17)]. For the western Pacific and Arabian Sea, using $\mathrm{SH}_{S}^{\overline{\mathrm{MLD}}}$ for the surface elevation within Eq. (17) results in lower error magnitudes across the time series. The differences in geostrophic time-averaged error for the western Pacific and Arabian Sea are 13 and $7 \mathrm{~cm} \mathrm{~s}^{-1}$, respectively. Corresponding percent decreases in error are $28 \%$ and $24 \%$. In the Gulf of Mexico, however, the same substitution results in larger error magnitudes. The time-averaged difference in error is $3 \mathrm{~cm} \mathrm{~s}^{-1}$, which corresponds with a $12 \%$ increase. Finally, evaluating Eq. (15) using $\mathrm{SH}_{S}^{\overline{\mathrm{MLD}}}$ results in much higher error magnitudes in all three regions compared with using the simple geostrophic constraint [Eq. (17)].

These conflicting results are explained by understanding the effects internal waves have on SSH. Equation (15) assumes a balance of velocity tendency, advection, Coriolis acceleration, and pressure gradient in the surface plane. In the western Pacific, strong internal tides impose a signature on $\mathrm{SSH}$ that is not well represented by the terms in Eqs. (15) and (17). Figure 16a shows western Pacific small-scale surface velocity while Fig. 16b shows small-scale surface vorticity. In this region, geostrophic velocity derived using small-scale SSH has both eddy and wave signatures throughout (Fig. 16c). Because of this, the resulting geostrophic vorticity is noisy with large magnitude wave interference patterns superimposed on the eddy field throughout (Fig. 16d). Using $\mathrm{SH}_{S}^{\overline{\mathrm{MLD}}}$ for the surface elevation term reduces the effects of internal waves. Low vertical mode, large amplitude internal waves primarily affect thermocline variability and have limited impact on the mixed layer. Integrating through only the MLD mutes the effect this phenomenon has on the surface elevation. This is evident in the 


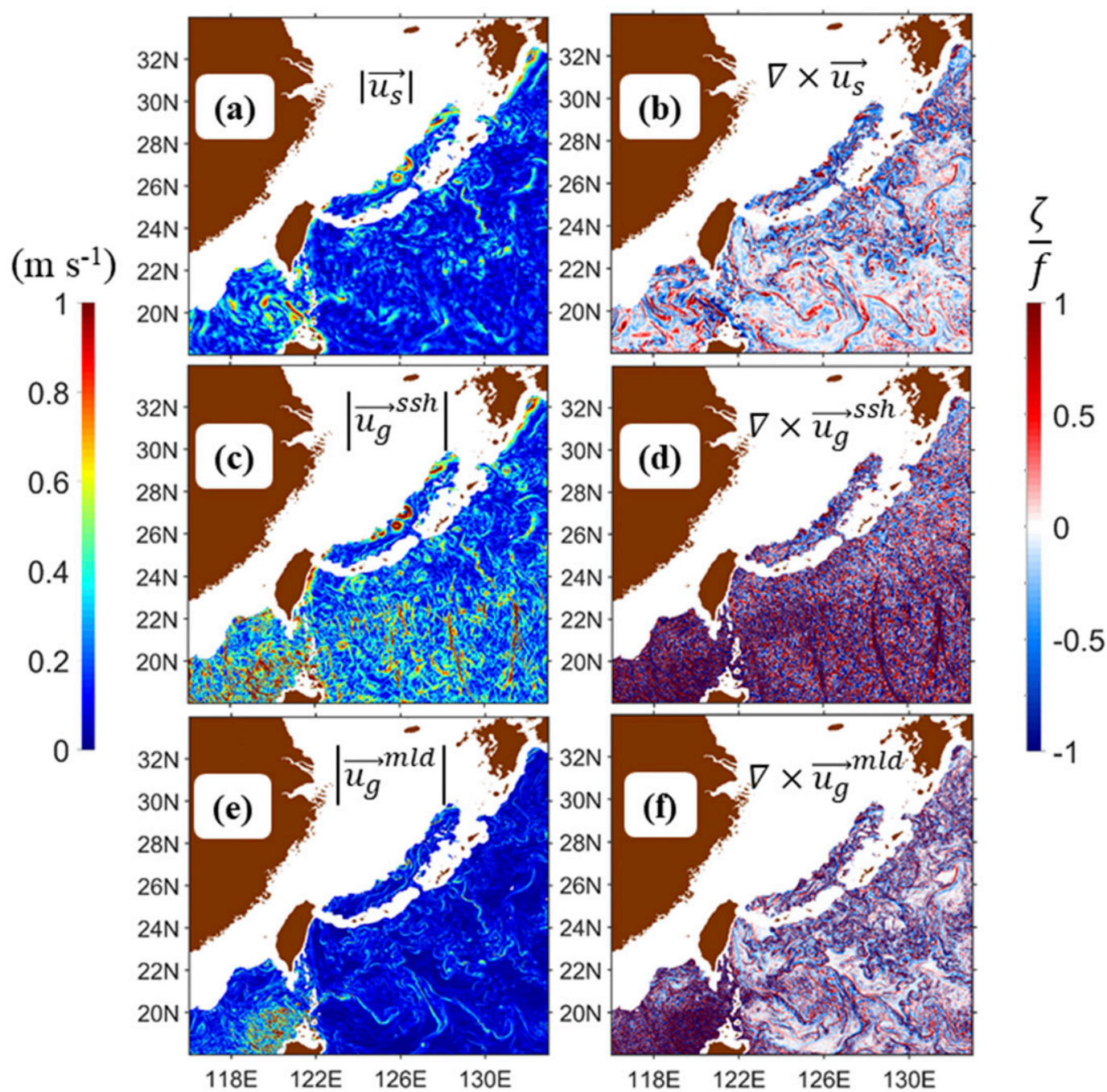

FIG. 16. Example of western Pacific small-scale (a) surface speed, (b) surface vorticity, (c) surface geostrophic speed calculated using small-scale sea surface height (SSH), (d) surface geostrophic vorticity calculated using smallscale SSH, (e) surface geostrophic speed calculated using small-scale SVA integrated through the area-averaged MLD, and (f) surface geostrophic vorticity calculated using small-scale SVA integrated through the area-averaged MLD. All vorticities have been normalized by the Coriolis parameter. Note the difference in color and range between the left and right columns.

geostrophic velocity derived using $\mathrm{SH}_{S}^{\overline{\mathrm{MLD}}}$ (Fig. 16e). The surface velocity field is much smoother and no wave signatures are apparent, a notable exception being the South China Sea where the area-averaged mixed layer is deeper than the local MLD and internal wave signatures have entered the analysis. The resulting vorticity (Fig. 16f) is much less noisy, with features that compare favorably with the true small-scale vorticity. Therefore, in the western Pacific, $\mathrm{SH}_{S}^{\overline{\mathrm{MLD}}}$ is a better representation of surface elevation because small-scale SSH is "contaminated" by strong internal wave dynamics.

For the Gulf of Mexico, Fig. 17a shows the small-scale surface velocity and Fig. 17b shows the small-scale surface vorticity. In comparison, Fig. $17 \mathrm{c}$ shows the small-scale surface geostrophic velocity derived using small-scale SSH. The field is dominated by eddy features and large magnitude wave signatures are not as readily identifiable as they were in the western Pacific. The corresponding geostrophic vorticity field is less noisy than the western Pacific equivalent, with clearly identifiable eddy features spread throughout (Fig. 17d). Geostrophic velocities calculated using $\mathrm{SH}_{S}^{\mathrm{MLD}}$ are smooth with fronts and eddies present throughout (Fig. 17e), but the eddy features have much lower magnitudes when compared with the true small-scale current. Likewise, the corresponding geostrophic vorticity 


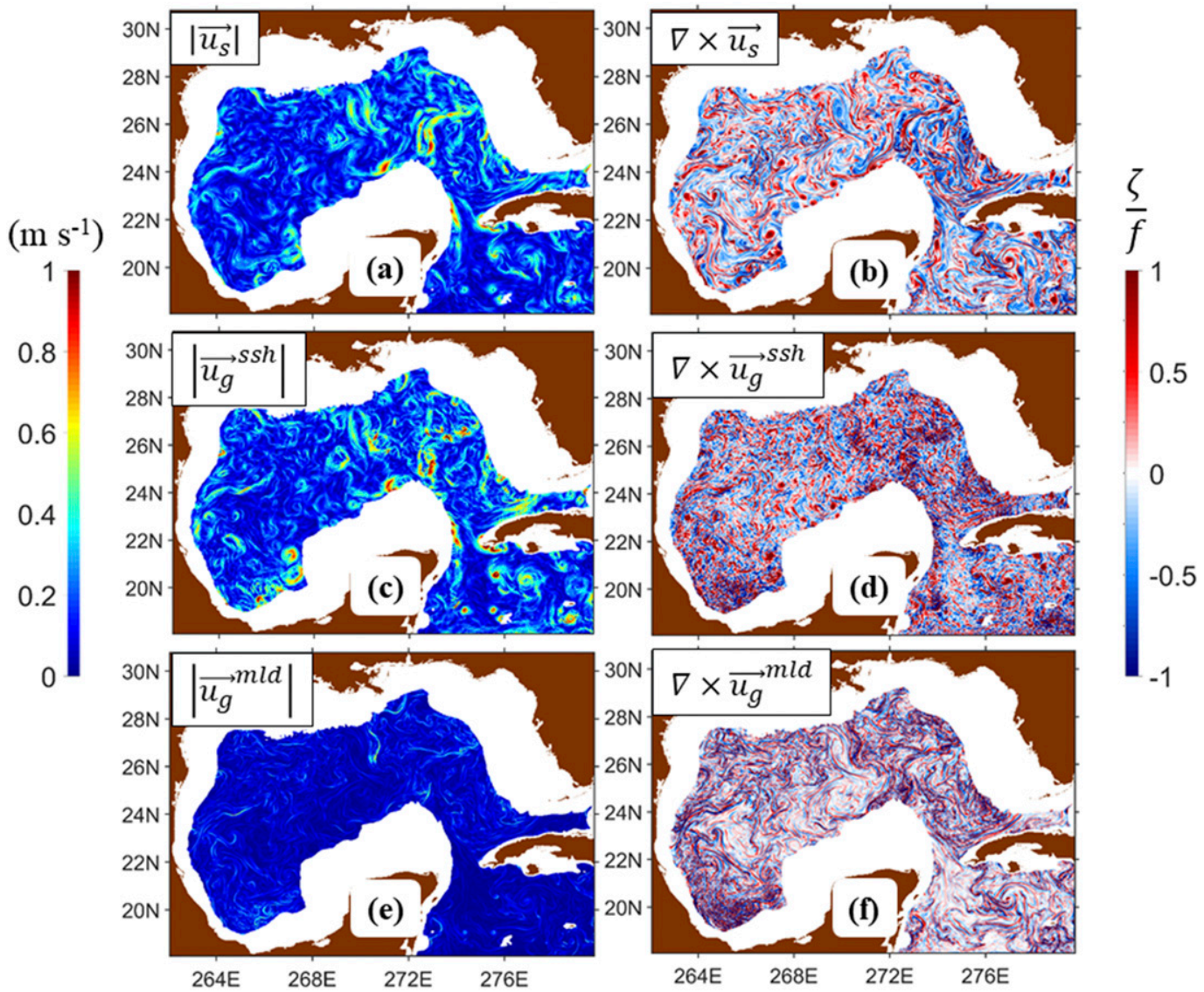

FIG. 17. Example of Gulf of Mexico small-scale (a) surface speed, (b) surface vorticity, (c) surface geostrophic speed calculated using small-scale sea surface height (SSH), (d) surface geostrophic vorticity calculated using smallscale SSH, (e) surface geostrophic speed calculated using small-scale SVA integrated through the area-averaged MLD, and (f) surface geostrophic vorticity calculated using small-scale SVA integrated through the area-averaged MLD. All vorticities have been normalized by the Coriolis parameter. Note the difference in color and range between the left and right columns.

field (Fig. 17f) has many corresponding features when compared with the true small-scale vorticity, but magnitudes do not consistently compare favorably. Thus, small-scale SSH is more representative of the pressure driving submesoscale eddies. This suggests that $\mathrm{SH}_{S}^{\overline{\mathrm{MLD}}}$ is not the most representative small-scale surface elevation term, but that it is advantageous in regions with strong baroclinic tides because smallscale SSH in these regions is heavily influenced by internal wave signatures.

Finally, the Arabian Sea results are more closely comparable to those from the western Pacific due to the presence of strong tidal forcing. Figures $18 \mathrm{a}$ and $18 \mathrm{~b}$ show the small-scale surface velocity and small-scale surface vorticity, respectively. Geostrophic velocities calculated using small-scale SSH (Fig. 18c) show a combination of eddy and wave motions, particularly in the southern half of the domain where wave features are more prevalent. As was observed in the western Pacific, the corresponding geostrophic vorticity is very noisy because of the entangled eddy and wave signatures (Fig. 18d). Substituting $\mathrm{SH}_{S}^{\overline{\mathrm{MLD}}}$ for smallscale $\mathrm{SSH}$, the geostrophic velocities become much smoother and more closely resemble the eddy motions noted in the true small-scale surface velocities (Fig. 18e). Likewise, the corresponding geostrophic vorticity (Fig. 18f) has features that clearly resemble those present in the true small-scale vorticity. Some areas in the south of the domain show the influence of internal wave motions due to local mixed layers that are shallower than the area-averaged MLD used in the integration of Eq. (18).

An additional unanswered question is why including tendency and advection in conjunction with $\mathrm{SH}_{S}^{\overline{\mathrm{MLD}}}$ produces higher error than the simple geostrophic approximation (Fig. 15; solid red lines versus dashed red 


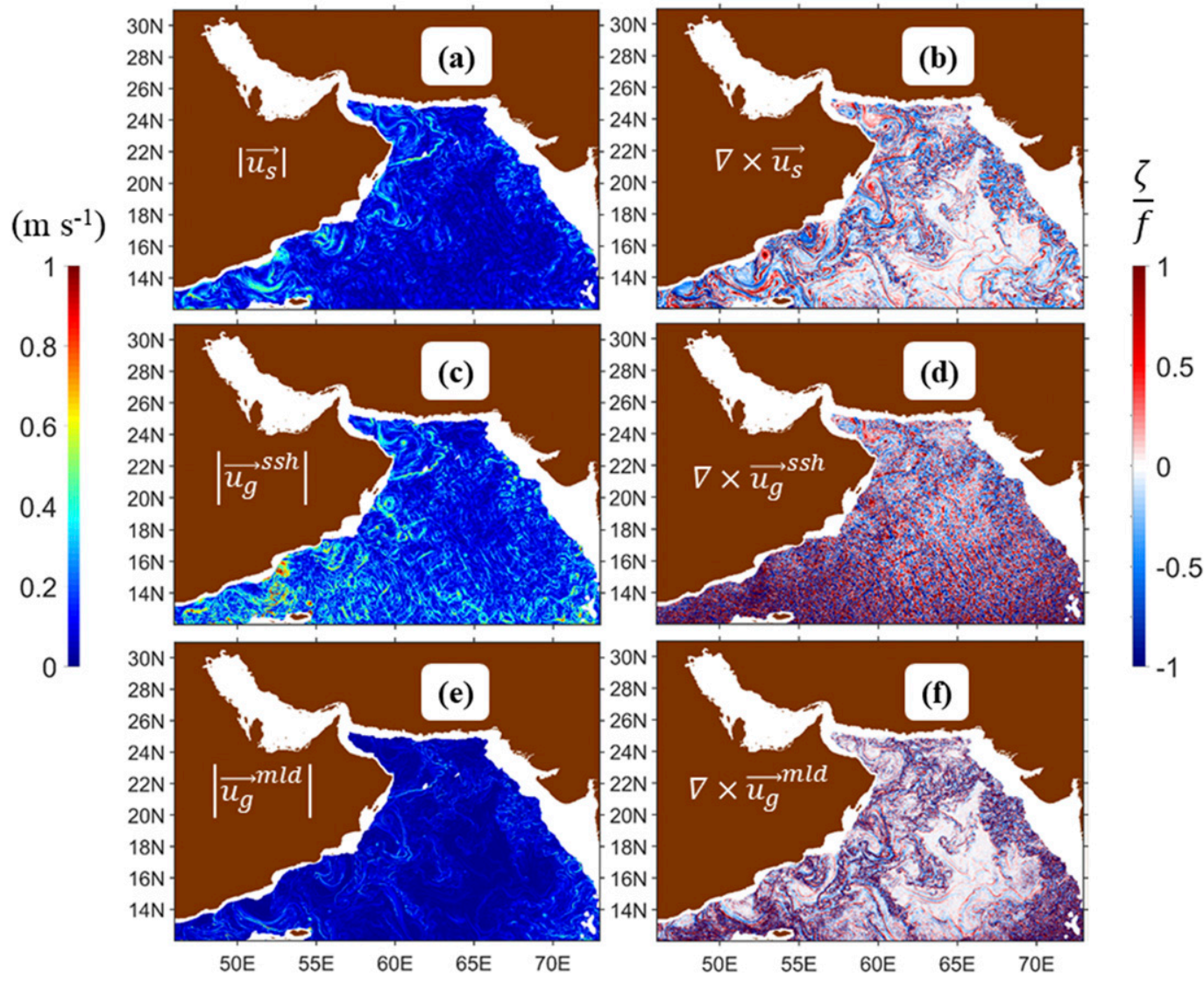

FIG. 18. Example of Arabian Sea small-scale (a) surface speed, (b) surface vorticity, (c) surface geostrophic speed calculated using small-scale SSH, (d) surface geostrophic vorticity calculated using small-scale SSH, (e) surface geostrophic speed calculated using small-scale SVA integrated through the area-averaged MLD, and (f) surface geostrophic vorticity calculated using small-scale SVA integrated through the area-averaged MLD. All vorticities have been normalized by the Coriolis parameter. Note the difference in color and range between the left and right columns.

lines). The primary information that $\mathrm{SH}_{S}^{\overline{\mathrm{MLD}}}$ lacks is the contribution of internal waves on surface elevation. This suggests that the small-scale velocities are a function of internal wave variability, and excluding the phenomenon from the surface elevation produces a less accurate dynamical constraint. Figure 19 supports this notion. In the western Pacific, both zonal and meridional small-scale velocity tendency have wave like features throughout. In the Arabian Sea, internal tide motions can clearly be seen in both the zonal and meridional velocity tendency. These results suggest that internal wave contributions to surface elevation partially balance the small-scale velocity tendency term. In the Gulf of Mexico, the velocity tendency is dominated by eddy features due to the weak tidal forcing in the area. Despite the lack of strong wave interaction, using $\mathrm{SH}_{S}^{\overline{\mathrm{MLD}}}$ in Eq. (15) in the Gulf of Mexico still results in the highest error solution (Fig. 15). This suggests that the small-scale SSH also more effectively balances the advective terms.
To conclude, we make a final observation and analysis. In Figs. 16-18, velocity and vorticity calculated using $\mathrm{SH}_{S}^{\overline{\mathrm{MLD}}}$ tended to produce smoother fields that better corresponded with the true smallscale velocity and vorticity. Differences in magnitude, however, were apparent, especially within strongly eddying motions. Figure 20a shows surface vorticity of a cyclonic submesoscale eddy in the Arabian Sea during January. Figure 20b shows the geostrophic vorticity using small-scale SSH over the same area. The eddy and the surrounding features can clearly be inferred, but the field is noisy, as was the case for the full field Arabian Sea analysis (Fig. 18). Figure 20d shows the resulting geostrophic vorticity when integrating small-scale SVA through the MLD. The field is smooth with many of the features showing strong correspondence with the true small-scale surface vorticity in location and magnitude. The primary exception being the eddy itself, substantial portions 


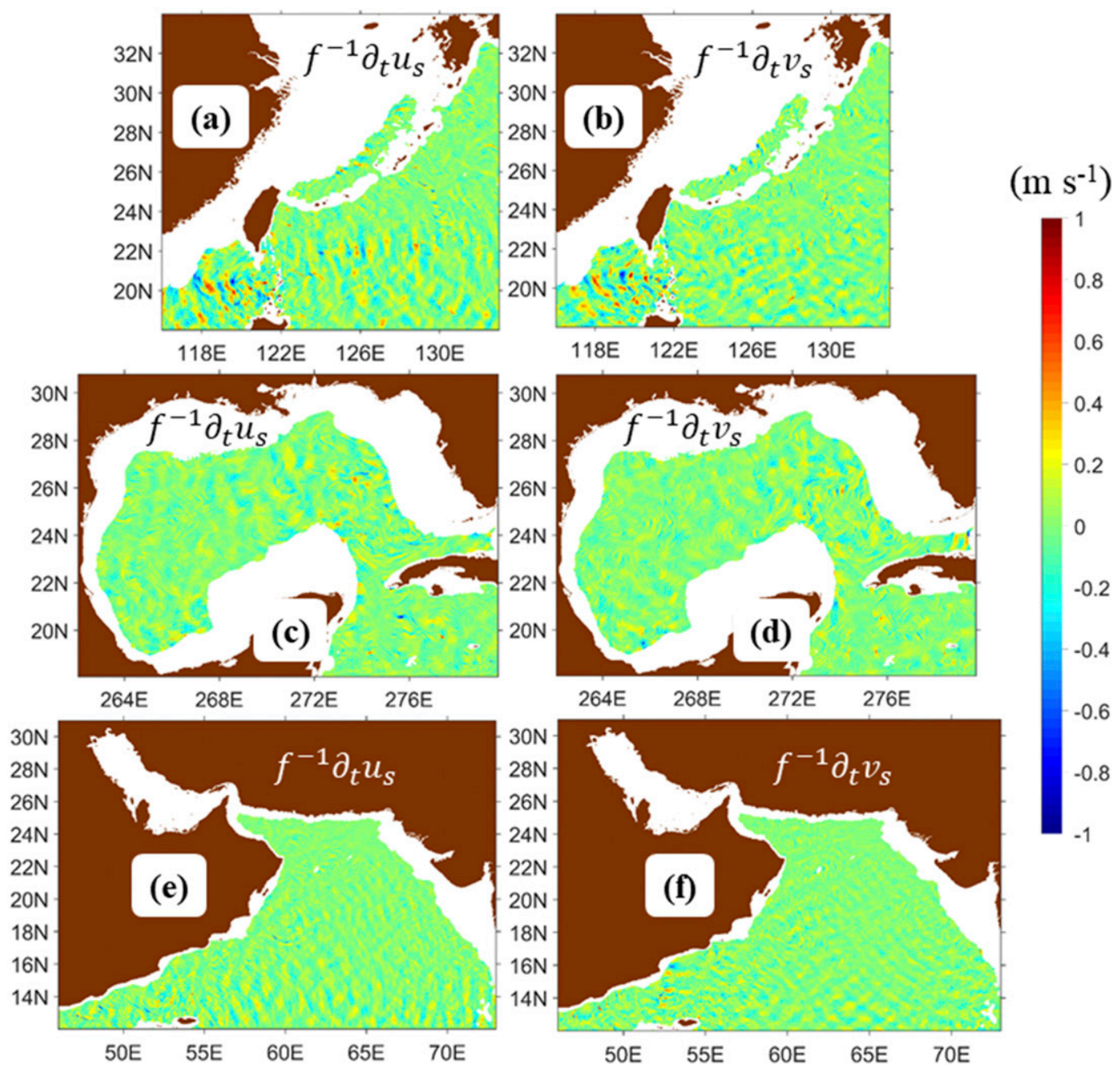

FIG. 19. Example of small-scale surface (left) zonal and (right) meridional velocity tendency normalized by the Coriolis parameter for (a),(b) the western Pacific, (c),(d) Gulf of Mexico, and (e),(f) Arabian Sea.

of which are rotating in the wrong direction. Why this is the case is clearly visible in Fig. 20c. Integrating only through the mixed layer, $\mathrm{SH}_{S}^{\overline{\mathrm{MLD}}}$ produces a positive (anticyclonic) surface elevation at the center of the eddy. Only by integrating the small-scale SVA deeper into the water column does the surface elevation begin to have the correct sign, and thus direction of rotation of the eddy. Figure 20e shows the resulting geostrophic vorticity when integrating $\mathrm{SH}_{S}$ to $800 \mathrm{~m}$ : the depth level at which $\mathrm{SH}_{S}$ and small-scale $\mathrm{SSH}$ are approximately the same. Vorticity within the eddy is now more comparable to the vorticity obtained when using small-scale SSH (Fig. 20b). The remainder of the surrounding field, however, no longer compares favorably with the true small-scale vorticity. This analysis suggests that the surrounding frontal features have surface small-scale velocity signatures that correspond with pressure signals within the mixed layer, while submesoscale eddies have essential baroclinic effects below the mixed layer.

\section{Summary and conclusions}

High-resolution simulations of three unique subregions of the global ocean have illuminated a robust set of statistics on the physical characteristics of submesoscale eddies. A summary of those results, in the order of their prior presentation, is given below:

- In each region, the majority of small-scale eddies have relatively low $\mathrm{SSH}$ amplitude $(\leq 3 \mathrm{~cm})$, though the cyclonic small-scale eddy probability distributions have relatively broad tails in which larger magnitude $(\geq 10 \mathrm{~cm})$ events can occur. The shallow tails in the anticyclonic small-scale eddy vorticity histograms are consistent with expectations of an instability in the anticyclonic flow when negative relative vorticity is 


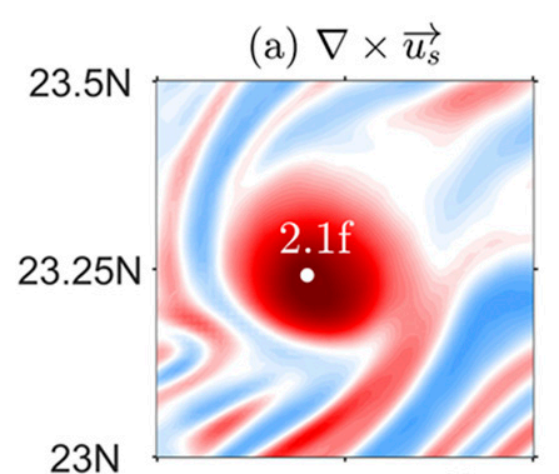

(d) $\nabla \times \vec{u}_{g}^{m l d}$

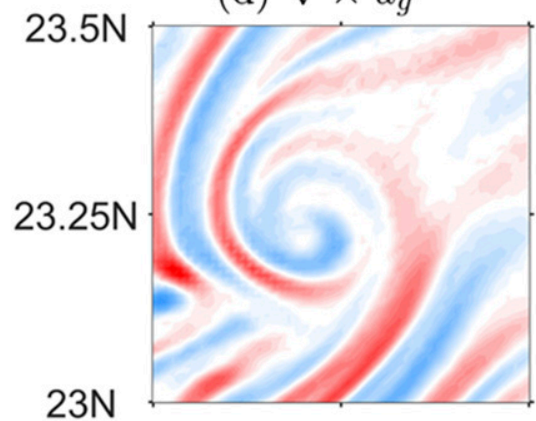

$23 \mathrm{~N}$ $60 \mathrm{E}$

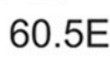

$60.5 \mathrm{E}$

$60.25 \mathrm{E}$

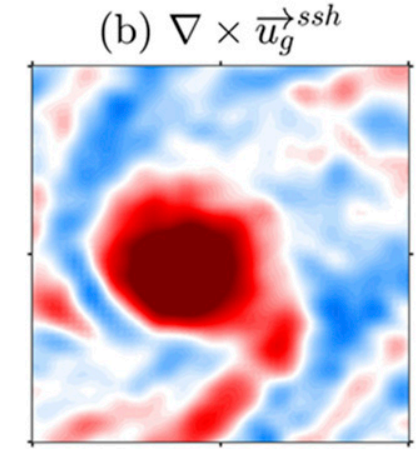

(e) $\nabla \times \vec{u}_{g}^{800 m}$

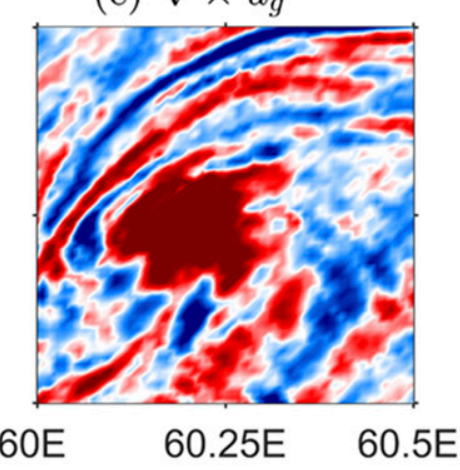

$\frac{\zeta}{f}-2$

$-1$

0

1

2

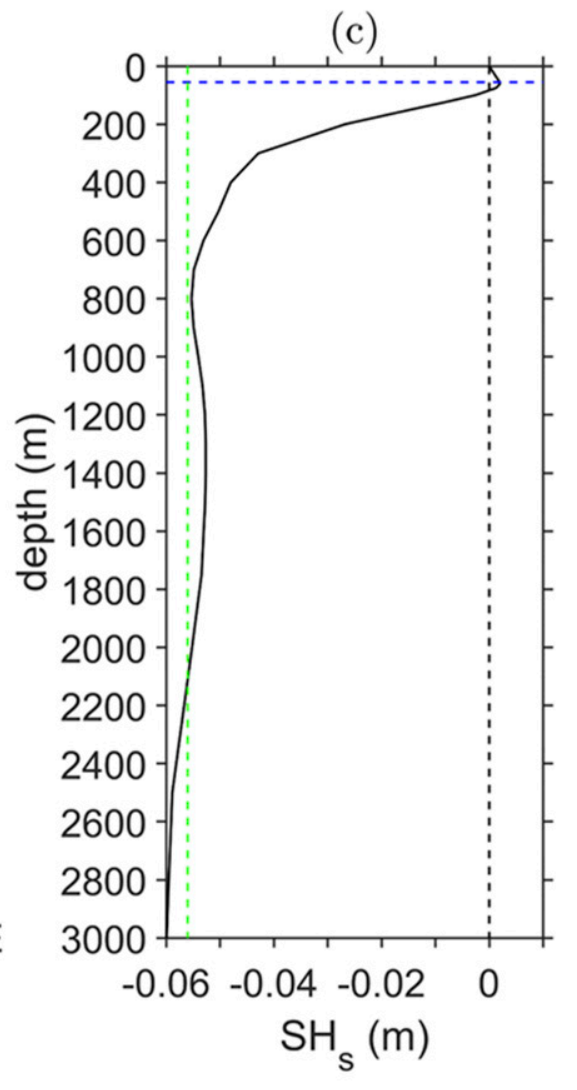

FIG. 20. Example submesoscale eddy in the Arabian Sea during January. (a) True small-scale surface vorticity. (b) Small-scale surface geostrophic vorticity calculated using small-scale SSH. (c) Progressive depth integration of small-scale SVA to generate small-scale steric height $\mathrm{SH}_{S}$ at the location of the small-scale SSH minimum labeled by a white dot in (a). The dashed blue line is the MLD, the dashed green line is the local small-scale SSH value, and the dashed black line is the zero line. (d) Small-scale surface geostrophic vorticity calculated using small-scale steric height integrated through the mixed layer. (e) Small-scale surface geostrophic vorticity calculated using the small-scale steric height integrated through $800 \mathrm{~m}$. All vorticities have been normalized by the Coriolis parameter.

greater than planetary vorticity. The number of cyclonic submesoscale eddies dominates the number of anticyclonic submesoscale eddies. Submesoscale eddy count is strongly correlated with the depth of the areaaveraged mixed layer.

- Large-scale vertical covariances of temperature, salinity, and SVA were found to have strong magnitudes within a near-surface layer and a separate deeper layer. In the deeper layer, strong off-diagonal elements suggest that large-scale temperature correlates over hundreds of meters in the vertical. Submesoscale eddy covariances have a much different depth-depth structure. Submesoscale SVA covariances are strong over a shallow region below the near-surface layer but have weak off-diagonal elements. This suggests that the submesoscale eddy phenomenon is more vertically localized and SVA anomalies are concentrated at and slightly below the base of a shallow near-surface layer.

- Mean cyclonic and anticyclonic submesoscale eddy SVA anomaly profiles are mirrored with the former having negative anomalies and the latter having positive anomalies. The mean anticyclonic submesoscale anomalies have largest magnitude right at the base of the mixed layer. Cyclonic submesoscale eddies are associated with isopycnals displaced upward, displacing cold water surfaceward, and leading to the negative SVA anomalies becoming maximal at approximately 2 times the MLD.

- Using small-scale SSH for surface elevation in conjunction with the small-scale velocity tendency and advection terms within the proposed dynamical constraint [Eq. (15)] produced lower error with respect to the submesoscale eddy velocity $(\sim 20 \%$ decrease) than assuming geostrophy [Eq. (17)]. The analysis was repeated, replacing small-scale SSH with the areaaveraged MLD integrated small-scale steric height. This largely removed the influence that strong internal wave motions have on surface elevation. For the western Pacific and Arabian Sea, this substitution resulted in lower overall errors ( $\geq 24 \%$ decrease) than 
when using small-scale SSH. In the Gulf of Mexico, using the small-scale SSH produced smaller errors (12\% lower). This is due to the weak barotropic, and thus baroclinic, tides in the Gulf of Mexico. In the western Pacific and Arabian Sea, tidal forcing is strong, and the resulting internal waves imprint strongly on SSH. When using the MLD integrated small-scale steric height for surface elevation, including tendency and advection produced larger errors than when assuming geostrophy. This is largely because small-scale velocity tendency is a function of internal wave variability. Therefore, small-scale SSH, which includes the contributions from internal wave motions, partially balances the small-scale velocity tendency term. Mixed layer integrated small-scale steric height, however, does not because it explicitly excludes large magnitude contributions to surface elevation due to internal wave motions. Finally, we found that the majority of the small-scale field is well represented by the MLD integrated small-scale steric height. However, submesoscale eddies, specifically, consistently had either the wrong magnitude, rotation, or both. Our analysis suggests that submesoscale eddies have significant baroclinic effects below the mixed layer.

From the perspective of ocean prediction, these results have great utility. For data assimilation systems that rely on a static background error covariance, submesoscale surface observations must be converted into subsurface corrections to the ocean model through vertical covariances like those presented here. Those corrections must then be subjected to a dynamical constraint in order to accurately spread local information to surrounding regions over submesoscale eddy length scales. The statistics developed here provide a first insight into what those vertical covariances and dynamical constraints might look like in an analysis/forecast system. They can also be used to validate data assimilation systems that extend covariance structure based on the model physics (e.g., 4DVAR). Nonetheless, the results are derived from numerical simulations and therefore need to be verified with observations of the real ocean. There currently exist no submesoscale observations over geographic areas and lengths of time comparable to those analyzed in this modeling study. The SWOT mission is expected to greatly help overcome this current observation deficit. Forthcoming SWOT calibration/validation efforts will test the accuracy of the SWOT data by collecting in situ data underneath fast repeat orbits (Wang et al. 2018). These experiments will likely prove invaluable for more definitively understanding the connection between submesoscale eddy surface expressions and their underlying depth structure.
Acknowledgments. This research is funded by the Naval Research Laboratory base program Submesoscale Prediction of Eddies by Altimeter Retrieval (SPEAR). This paper is contribution NRL/JA-7320-19-4383 and has been approved for public release. The authors thank Pete Spence for producing and providing the Gulf of Mexico simulations. The authors are also grateful for two anonymous reviewers whose helpful suggestions improved the manuscript.

\section{REFERENCES}

Bachman, S. D., J. R. Taylor, K. A. Adams, and P. J. Hosegood, 2017: Mesoscale and submesoscale effects on mixed layer depth in the Southern Ocean. J. Phys. Oceanogr., 47, 21732188, https://doi.org/10.1175/JPO-D-17-0034.1.

Barron, C. N., and L. F. Smedstad, 2002: Global river inflow within the Navy Coastal Ocean Model. Proc. Oceans '02 MTS/IEEE, Biloxi, MS, IEEE, 1472-1479, https://doi.org/ 10.1109/OCEANS.2002.1191855.

_ A. B. Kara, P. J. Martin, R. C. Rhodes, and L. F. Smedstad, 2006: Formulation, implementation and examination of vertical coordinates choices in the Global Navy Coastal Ocean Model (NCOM). Ocean Modell., 11, 347-375, https://doi.org/ 10.1016/j.ocemod.2005.01.004.

Boccaletti, G., R. Ferrari, and B. Fox-Kemper, 2007: Mixed layer instabilities and restratification. J. Phys. Oceanogr., 37, 22282250, https://doi.org/10.1175/JPO3101.1.

Brannigan, L., 2016: Intense submesoscale upwelling in anticyclonic eddies. Geophys. Res. Lett., 43, 3360-3369, https:// doi.org/10.1002/2016GL067926.

_ D. P. Marshall, A. C. N. Garabato, A. J. George Nurser, and J. Kaiser, 2017: Submesoscale instabilities in mesoscale eddies. J. Phys. Oceanogr., 47, 3061-3085, https://doi.org/10.1175/ JPO-D-16-0178.1.

Callies, J., R. Ferrari, J. M. Klymak, and J. Gula, 2015: Seasonality in submesoscale turbulence. Nat. Commun., 6, 6862, https:// doi.org/10.1038/ncomms7862.

Capet, X., J. C. McWilliams, M. J. Molemaker, and A. F. Shchepetkin, 2008a: Mesoscale to submesoscale transition in the California Current system. Part I: Flow structure, eddy flux, and observational tests. J. Phys. Oceanogr., 38, 29-43, https://doi.org/10.1175/2007JPO3671.1.

,,-- , and,$- 2008 \mathrm{~b}$ : Mesoscale to submesoscale transition in the California Current system. Part II: Frontal processes. J. Phys. Oceanogr., 38, 29-43, https://doi.org/ 10.1175/2007JPO3672.1.

Chang, Y. S., B.-J. Choi, and Y.-G. Park, 2018: Seasonal variation of submesoscale flow features in a mesoscale eddy-dominate region in the East Sea. Ocean Sci. J., 53, 191-206, https:// doi.org/10.1007/s12601-018-0008-0.

Chavanne, C., P. Flament, D. Luther, and K.-W. Gurgel, 2010: The surface expression of semidiurnal internal tides near a strong source at Hawaii. Part II: Interactions with mesoscale currents. J. Phys. Oceanogr., 40, 1180-1200, https://doi.org/ 10.1175/2010JPO4223.1.

Chelton, D. B., R. A. DeSzoeke, G. Schlax, K. E. Naggar, and N. Siwertz, 1998: Geographical variability of the first baroclinic Rossby radius of deformation. J. Phys. Oceanogr., 28, 433-460, https://doi.org/10.1175/1520-0485(1998)028<0433: GVOTFB $>2.0 . \mathrm{CO} ; 2$. 
Cummings, J. A., 2005: Operational multivariate ocean data assimilation. Quart. J. Roy. Meteor. Soc., 131, 3583-3604, https:// doi.org/10.1256/qj.05.105.

— , and Coauthors, 2009: Ocean data assimilation systems for GODAE. Oceanography, 22, 96-109, https://doi.org/10.5670/ oceanog.2009.69.

D'Addezio, J. M., S. Smith, G. A. Jacobs, R. Helber, C. Rowley, I. Souopgui, and M. J. Carrier, 2019: Quantifying wavelengths constrained by simulated SWOT observations in a submesoscale resolving ocean analysis/forecasting system. Ocean Modell., 135, 40-55, https://doi.org/10.1016/j.ocemod.2019.02.001.

D'Asaro, E. A., and Coauthors, 2018: Ocean convergence and dispersion of flotsam. Proc. Natl. Acad. Sci. USA, 115, 1162 1167, https://doi.org/10.1073/pnas.1718453115.

Ducet, N., P. Y. Le Traon, and G. Reverdin, 2000: Global highresolution mapping of ocean circulation from TOPEX/Poseidon and ERS-1 and -2. J. Geophys. Res., 105, 19477-19498, https:// doi.org/10.1029/2000JC900063.

Egbert, G. D., and S. Y. Erofeeva, 2002: Efficient inverse modeling of barotropic ocean tides. J. Atmos. Oceanic Technol., 19, 183-204, https://doi.org/10.1175/1520-0426(2002)019<0183: EIMOBO $>2.0 . \mathrm{CO} ; 2$.

Fu, L. L., and C. Ubelmann, 2014: On the transition from profile altimetry to swath altimeter for observing global ocean surface topography. J. Atmos. Oceanic Technol., 31, 560-568, https:// doi.org/10.1175/JTECH-D-13-00109.1.

Helber, R. W., A. B. Kara, J. G. Richman, M. R. Carnes, C. N. Barron, H. E. Hurlburt, and T. Boyer, 2012: Temperature versus salinity gradients below the ocean mixed layer. J. Geophys. Res., 117, C05006, https://doi.org/10.1029/ 2011JC007382.

—, T. L. Townsend, C. N. Barron, J. M. Dastugue, and M. R. Carnes, 2013: Validation test report for the Improved Synthetic Ocean Profile (ISOP) system, Part I: Synthetic profile methods and algorithm. NRL Tech. Rep. NRL/MR/7320--13-9364, 128 pp., https://apps.dtic.mil/dtic/tr/fulltext/u2/a585251.pdf.

Henson, S. A., and A. C. Thomas, 2008: A census of oceanic anticyclonic eddies in the Gulf of Alaska. Deep-Sea Res. I, 55, 163-176, https://doi.org/10.1016/j.dsr.2007.11.005.

Hodur, R. M., 1997: The Naval Research Laboratory's Coupled Ocean/Atmosphere Mesoscale Prediction System (COAMPS). Mon. Wea. Rev., 125, 1414-1430, https://doi.org/10.1175/15200493(1997)125<1414:TNRLSC $>2.0$. CO;2.

Hogan, T. F., and Coauthors, 2014: The Navy Global Environmental Model. Oceanography, 27, 116-125, https://doi.org/10.5670/ oceanog.2014.73.

Hoskins, B. J., 1982: The mathematical theory of frontogenesis. Annu. Rev. Fluid Mech., 14, 131-151, https://doi.org/10.1146/ annurev.fl.14.010182.001023.

, and F. P. Bretherton, 1972: Atmospheric frontogenesis models: Mathematical formulation and solution. J. Atmos. Sci., 29, 11-37, https://doi.org/10.1175/1520-0469(1972)029<0011: AFMMFA $>2.0 . \mathrm{CO} ; 2$.

Hua, B. L., and P. Klein, 1998: An exact criterion for the stirring properties of nearly two-dimensional turbulence. Physica $D$, 113, 98-110, https://doi.org/10.1016/S0167-2789(97)00143-7.

Isern-Fontanet, J., E. Garcia-Ladona, and J. Font, 2003: Identification of marine eddies from altimetric maps. J. Atmos. Oceanic Technol., 20, 772-778, https://doi.org/10.1175/1520-0426(2003) $20<772$ :IOMEFA $>2.0 . \mathrm{CO} ; 2$.

Jacobs, J. A., J. G. Richman, J. D. Doyle, P. L. Spence, B. P. Bartels, C. N. Barron, R. W. Helber, and F. L. Bub, 2014: Simulating conditional deterministic predictability within ocean frontogenesis. Ocean Modell., 78, 1-16, https://doi.org/ 10.1016/j.ocemod.2014.02.004.

Klein, P., and G. Lapeyre, 2009: The oceanic vertical pump induced by mesoscale and submesoscale turbulence. Annu. Rev. Mar. Sci., $\mathbf{1}$, 351-375, https://doi.org/10.1146/annurev.marine.010908.163704.

Koszalka, I., A. Bracco, J. C. McWilliams, and A. Provenzale, 2009: Dynamics of wind-forced coherent anticyclones in the open ocean. J. Geophys. Res., 114, C08011, https://doi.org/10.1029/ 2009JC005388.

Kurian, J., F. Colas, X. Capet, J. C. McWilliams, and D. B. Chelton, 2011: Eddy properties in the California Current System. J. Geophys. Res., 116, C08027, https://doi.org/ 10.1029/2010JC006895.

Levine, M. D., 1983: Internal waves in the ocean: A review. Rev. Geophys., 21, 1206-1216, https://doi.org/10.1029/RG021i005p01206.

Lévy, M., R. Ferrari, P. J. S. Franks, A. P. Martin, and P. Riviere, 2012: Bringing physics to life at the submesoscale. Geophys. Res. Lett., 39, L14602, https://doi.org/10.1029/2012GL052756.

_ , P. J. S. Franks, and K. Shafer Smith, 2018: The role of submesoscale currents in structuring marine ecosystems. Nat. Commun., 9, 4758, https://doi.org/10.1038/s41467-018-07059-3.

Li, Z., J. C. McWilliams, K. Ide, and J. D. Farrara, 2015a: A multiscale variational data assimilation scheme: Formulation and illustration. Mon. Wea. Rev., 143, 3804-3822, https://doi.org/ 10.1175/MWR-D-14-00384.1.

,,--- , and $-2015 \mathrm{~b}$ : Coastal ocean data assimilation using a multi-scale three-dimensional variational scheme. Ocean Dyn., 65, 1001-1015, https://doi.org/10.1007/s10236015-0850-x.

Lynett, P. J., and P. L.-F. Liu, 2002: A two-dimensional, depth integrated model for internal wave propagation over variable bathymetry. Wave Motion, 36, 221-240, https://doi.org/ 10.1016/S0165-2125(01)00115-9.

McWilliams, J. C., 2016: Submesoscale currents in the ocean. Proc. Roy. Soc., 472A, 20160117, https://doi.org/10.1098/rspa.2016.0117.

Metzger, E. J., and Coauthors, 2017: Global ocean forecast system 3.1 validation test. NRL Tech. Rep. NRL/MR/7320-17-9722, $60 \mathrm{pp}$, https://apps.dtic.mil/dtic/tr/fulltext/u2/1034517.pdf.

Morvan, M., P. L'Hegaret, X. Carton, J. Gula, C. Vic, C. de Marez, M. Sokolovskiy, and K. Koshel, 2019: The life cycle of submesoscale eddies generated by topographic interactions. Ocean Sci., 15, 1531-1543, https://doi.org/10.5194/ os-15-1531-2019.

Okubo, A., 1970: Horizontal dispersion of floatable particles in the vicinity of velocity singularities such as convergences. Deep-Sea Res. Oceanogr. Abstr., 17, 445-454, https://doi.org/ 10.1016/0011-7471(70)90059-8.

Qiu, B., S. Chen, P. Klein, H. Sasaki, and Y. Sasai, 2014: Seasonal mesoscale and submesoscale eddy variability along the North Pacific Subtropical Countercurrent. J. Phys. Oceanogr., 44, 3079-3098, https://doi.org/10.1175/JPO-D-14-0071.1.

Richman, J. G., B. K. Arbic, J. F. Shriver, E. J. Metzger, and A. J. Wallcraft, 2012: Inferring dynamics from wavenumber spectra of an eddying global ocean model with embedded tides. J. Geophys. Res., 117, C12012, https://doi.org/10.1029/ 2012JC008364.

Rocha, C. B., S. T. Gille, T. K. Chereskin, and D. Menemenlis, 2016: Seasonality of submesoscale dynamics in the Kuroshio extension. Geophys. Res. Lett., 43, 11304-11311, https:// doi.org/10.1002/2016GL071349.

Shcherbina, A. Y., E. A. D'Asaro, C. M. Lee, J. M. Klymak, M. J. Molemaker, and J. C. McWilliams, 2013: Statistics of vertical vorticity, divergence, and strain in a developed submesoscale 
turbulence field. Geophys. Res. Lett., 40, 4706-4711, https:// doi.org/10.1002/grl.50919.

Shriver, J. F., J. G. Richman, and B. K. Arbic, 2014: How stationary are the internal tides in a high-resolution global ocean circulation model? J. Geophys. Res. Oceans, 119, 2769-2787, https://doi.org/10.1002/2013JC009423.

St. Laurent, L., M. H. Alford, and T. Paluszkiewicz, 2012: An introduction to the special issue on internal waves. Oceanography, 25, 15-19, https://doi.org/10.5670/oceanog.2012.37.

Su, Z., J. Wang, P. Klein, A. F. Thompson, and D. Menemenlis, 2018: Ocean submesoscale as a key component of the globa heat budget. Nat. Commun., 9, 775, https://doi.org/10.1038/ s41467-018-02983-w.

Thomas, L. N., and R. Ferrari, 2008: Friction, frontogenesis, and the stratification of the surface mixed layer. J. Phys. Oceanogr., 38, 2501-2518, https://doi.org/10.1175/2008JPO3797.1.

A. Tandon, and A. Mahadevan, 2008: Submesoscale processes and dynamics. Ocean Modeling in an Eddying Regime, Geophys. Monogr., Vol. 177, Amer. Geophys. Union, 17-38.

Thompson, A. F., and A. Lazar, 2016: Open-ocean submesoscale motions: A full seasonal cycle of mixed layer instabilities from gliders. J. Phys. Oceanogr., 46, 1285-1307, https://doi.org/ 10.1175/JPO-D-15-0170.1.

Wang, J., L.-L. Fu, B. Qiu, D. Menemenlis, J. T. Farrar, Y. Chao, A. F. Thompson, and M. M. Flexas, 2018: An observing system simulation experiment for the calibration and validation of the Surface Water Ocean Topography sea surface height measurement using in situ platforms. J. Atmos. Oceanic Technol., 35, 281-297, https://doi.org/10.1175/JTECH-D-17-0076.1.

Weiss, J., 1991: The dynamics of enstrophy transfer in two dimensional hydrodynamics. Physica D, 48, 273-294, https:// doi.org/10.1016/0167-2789(91)90088-Q.

Williams, S., M. Hecht, M. Petersen, R. Strelitz, M. Maltrud, J. Ahrens, M. Hlawitschka, and B. Hamann, 2011: Visualization and analysis of eddies in a global ocean simulation. Comput. Graph. Forum, 30, 991-1000, https://doi.org/10.1111/j.14678659.2011.01948.x.

Yaremchuk, M., M. Carrier, S. Smith, and G. Jacobs, 2013: Background error correlation modeling with diffusion operator. Data Assimilation for Atmospheric, Oceanic and Hydrologic Applications (Vol. II), S. Park and L. Xu, Eds., Springer, 177203, https://doi.org/10.1007/978-3-642-35088-7_8.

$\mathrm{Yu}$, X., A. C. Naveira Garabato, A. P. Martin, C. E. Buckingham, L. Brannigan, and Z. Su, 2019: An annual cycle of submesoscale vertical flow and restratification in the upper ocean. J. Phys. Oceanogr., 49, 1439-1461, https://doi.org/10.1175/ JPO-D-18-0253.1.

Zhong, Y., and A. Bracco, 2013: Submesoscale impacts on horizontal and vertical transport in the Gulf of Mexico. J. Geophys. Res. Oceans, 118, 5651-5668, https://doi.org/10.1002/jgrc.20402.

J. Tian, J. Dong, W. Zhao, and Z. Zhang, 2017: Observed and simulated submesoscale vertical pump of an anticyclonic eddy in the South China Sea. Sci. Rep., 7, 44011, https://doi.org/10.1038/srep44011. 